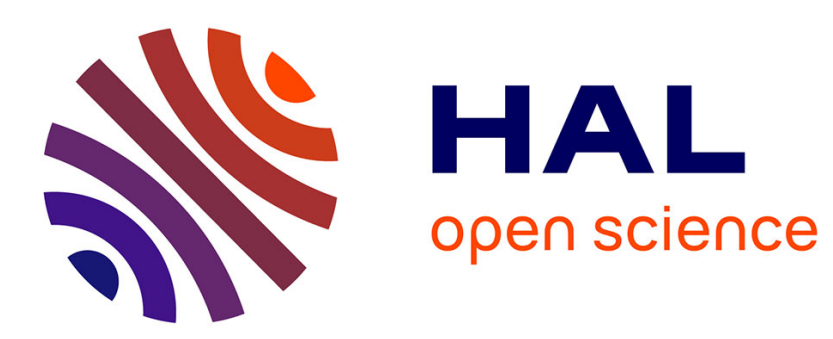

\title{
Image Segmentation Using Active Contours: Calculus of Variations or Shape Gradients?
}

Gilles Aubert, Michel Barlaud, Olivier Faugeras, Stéphanie Jehan-Besson

\section{To cite this version:}

Gilles Aubert, Michel Barlaud, Olivier Faugeras, Stéphanie Jehan-Besson. Image Segmentation Using Active Contours: Calculus of Variations or Shape Gradients?. SIAM Journal on Applied Mathematics, 2003, 63 (6), pp.2128-2154. 10.1137/S0036139902408928 . hal-00964649

\section{HAL Id: hal-00964649 \\ https://hal.science/hal-00964649}

Submitted on 24 Mar 2014

HAL is a multi-disciplinary open access archive for the deposit and dissemination of scientific research documents, whether they are published or not. The documents may come from teaching and research institutions in France or abroad, or from public or private research centers.
L'archive ouverte pluridisciplinaire HAL, est destinée au dépôt et à la diffusion de documents scientifiques de niveau recherche, publiés ou non, émanant des établissements d'enseignement et de recherche français ou étrangers, des laboratoires publics ou privés. 


\title{
IMAGE SEGMENTATION USING ACTIVE CONTOURS: CALCULUS OF VARIATIONS OR SHAPE GRADIENTS?*
}

\author{
GILLES AUBERT ${ }^{\dagger}$, MICHEL BARLAUD ${ }^{\ddagger}$, OLIVIER FAUGERAS $^{\S}$, AND \\ STÉPHANIE JEHAN-BESSON ${ }^{\ddagger}$
}

\begin{abstract}
We consider the problem of segmenting an image through the minimization of an energy criterion involving region and boundary functionals. We show that one can go from one class to the other by solving Poisson's or Helmholtz's equation with well-chosen boundary conditions. Using this equivalence, we study the case of a large class of region functionals by standard methods of the calculus of variations and derive the corresponding Euler-Lagrange equations. We revisit this problem using the notion of a shape derivative and show that the same equations can be elegantly derived without going through the unnatural step of converting the region integrals into boundary integrals. We also define a larger class of region functionals based on the estimation and comparison to a prototype of the probability density distribution of image features and show how the shape derivative tool allows us to easily compute the corresponding Gâteaux derivatives and Euler-Lagrange equations. Finally we apply this new functional to the problem of regions segmentation in sequences of color images. We briefly describe our numerical scheme and show some experimental results.
\end{abstract}

Key words. image segmentation, region segmentation, active contours, active regions, image statistics, region functionals, boundary functionals, calculus of variations, shape optimization, shape gradient, Euler-Lagrange equations, Gâteaux derivative, Parzen window estimation, level set methods

AMS subject classifications. 35, 35K, 49, 49Q10, 68U10

DOI. $10.1137 /$ S0036139902408928

1. Introduction. Many problems in image processing, such as segmentation, tracking, or classification, can be cast in the framework of optimization theory, e.g., as the minimization of some energy measure. The energy is often some combination of region or boundary functionals. The minimization is usually not trivial, and many methods have been developed to reach an optimum which may be only local.

We address here the problem of the optimization of region or boundary functionals with the method of active contours. Active contours have been introduced by Kass, Witkin and Terzopoulos [34] and were originally boundary methods. Snakes [34], balloons [10], or geodesic active contours [4] are driven towards the edges of an image through the minimization of a boundary integral of functions of features depending on edges. Active contours driven by region functionals in addition to boundary functionals have appeared later. Introduced by [11] and [43], they have been further developed in $[52,5,9,38,39,40,41,21,51]$ and $[31,33]$. In effect, the use of active contours for the optimization of a criterion including both region and boundary functionals appears to be really powerful.

In general, features of the image region to be segmented, tracked, etc.,... are embedded in region functionals while the boundary functional allows smoothness and

\footnotetext{
* Received by the editors May 29, 2002; accepted for publication (in revised form) January 29, 2003; published electronically September 17, 2003.

http://www.siam.org/journals/siap/63-6/40892.html

${ }^{\dagger}$ Laboratoire Dieudonné, CNRS-UNSA, Parc Valrose, 06108 Nice Cedex 2, France (gaubert@ math.unice.fr).

${ }^{\ddagger}$ Laboratoire I3S, CNRS-UNSA, 2000 route des Lucioles, 06903 Sophia Antipolis, France (barlaud @i3s.unice.fr, jehan@i3s.unice.fr).

$\S$ INRIA Sophia Antipolis, 2004 route des Lucioles, BP 93 06902, Sophia-Antipolis Cedex, France (faugeras@sophia.inria.fr).
} 
regularity of the region boundary. The basic principle is to construct a parabolic partial differential equation (PDE) from the energy criterion, e.g., by computing some sort of Euler-Lagrange equations; this PDE changes the shape of the current curve according to some velocity field which can be thought of as a direction of descent of the energy criterion. Given a closed curve enclosing an initial region one then computes the solution of this PDE for this initial condition. The corresponding family of curves decreases the energy criterion and converges toward a (local) minimum of the criterion hopefully corresponding to the objects to be segmented. To compute such a PDE, several methods have been proposed.

Some authors do not compute the theoretical expression of the velocity field (basically the gradient of the energy criterion) but choose a deformation of the curve that will make the criterion decrease [5,9] (they compute a direction of descent). Other authors $[52,39,41]$ compute the theoretical expression of the velocity vector from the Euler-Lagrange equations. The computation is performed in three main steps. First, region integrals representing region functionals are transformed into boundary integrals using the Green-Riemann theorem. Second, the corresponding Euler-Lagrange equations are derived and used to define a dynamic scheme to evolve the initial region. Another alternative is to keep the region formulation to compute the gradient of the energy criterion with respect to the region boundary instead of reducing region integrals to boundary integrals. In [21], the authors propose computing the derivative of the criterion while taking into account the discontinuities across the contour. In $[31,33]$ the computation of the evolution equation is achieved through shape derivation principles.

This computation becomes more involved when global information about regions is present in the energy criterion, the so-called region-dependent case. It happens, for example, when statistical features of a region such as, for example, the mean or the variance of the intensity are involved in the minimization. This case has been studied in $[6,7,20,21,31,33,51]$. In $[31,33]$ the authors show that the minimization of functionals involving region-dependent features induces additional terms in the evolution equation of the active contour that are important in practice. These additional terms are easily computed thanks to shape derivation tools.

In this article we clarify the relationships between the boundary and region functionals that arise naturally in several image processing tasks. We show in section 3 that one can go from one to the other by solving Poisson's equation with Dirichlet conditions or Helmholtz's equation with Neumann conditions.

We then concentrate on the problem of finding local minima of a large class of region functionals. In section 4 we first transform them into boundary functionals and apply methods from the calculus of variations to compute the corresponding Gâteaux derivatives and construct a velocity field on the region boundary. This field defines a PDE whose solution for a given initial boundary condition defines a oneparameter family of regions which, in practice, converges towards a local minimum of the functional. The problem of the existence and uniqueness of a solution to this $\mathrm{PDE}$ is not addressed in this article.

We next change our point of view and rederive the same equations in a simpler and more natural way, i.e., without going through the trouble of turning region integrals into boundary integrals; this is achieved in section 5 by applying shape derivation methods $[49,22]$. This line of approach has already been followed in [46] in his work on the estimation of the optical flow.

We then turn in section 6 our attention to a new class of region-based functionals 
by considering histograms of image features. The shape derivation tools allow us to easily derive the velocity field that defines the evolution of the region boundary.

Section 7 is devoted to an application of the previous methods to the problem of region segmentation with a given color histogram in a sequence of images. Our experimental results show that the technique indeed has some interesting potentials.

2. Problem statement. In many image processing problems, the issue is to find a set of image regions that minimize a given error criterion. This criterion is often a combination of region and boundary functionals.

A local minimizer for such a criterion including both region and boundary functionals is usually hard to compute. This is mostly due to the fact that the set of image regions, i.e., the set of regular open domains in $\mathbb{R}^{n}$ (whose boundary is a closed, $C^{2}$ manifold), does not have a structure of vector space, preventing us from using in a straightforward fashion gradient descent methods. In order to circumvent this difficulty, calculus of variations and shape optimization techniques can be brought to bear on the problem. The basic idea is to use them in order to derive a PDE that will drive the boundary of an initial region toward a local minimum of the error criterion. The key point is to compute the velocity vector at each point of the boundary at each time instant. In this paper we propose a framework for achieving these goals in a number of practically important cases.

To fix ideas in the two-dimensional case, the evolving boundary, or active contour, is modeled by a parametric curve $\Gamma(s, \tau)=\left(x_{1}(s, \tau), x_{2}(s, \tau)\right)$, where $s$ may be its arclength and $\tau$ is an evolution parameter - the time. The active contour is then driven by the following PDE:

$$
\Gamma_{\tau} \stackrel{\text { def }}{=} \frac{\partial \Gamma(s, \tau)}{\partial \tau}=\mathbf{v} \quad \text { with } \Gamma(\tau=0)=\Gamma_{0}
$$

where $\Gamma_{0}$ is an initial curve defined by the user and $\mathbf{v}$ the velocity vector of $\Gamma(s, \tau)$. This velocity is the unknown that must be derived from the error criterion so that the solution $\Gamma(., \tau)$ converges towards a curve achieving a local minimum and thus, hopefully, towards the boundary of the object to segment as $\tau \rightarrow \infty$.

2.1. Boundary and region functionals. Let us now define more precisely the region and boundary functionals. Let $\mathcal{U}$ be a class of domains (open, regular bounded sets, i.e., $C^{2}$ ) of $\mathbb{R}^{n}$ and $\Omega$ an element of $\mathcal{U}$ of boundary $\partial \Omega$, which we sometimes denote $\Gamma$. A boundary functional, $J_{b}$, may be expressed as a boundary integral of some scalar function $g$ of image features:

$$
J_{b}(\partial \Omega)=\int_{\partial \Omega} g(\mathbf{x}) d \mathbf{a}(\mathbf{x}),
$$

where $\partial \Omega$ is the boundary of the region and $d \mathbf{a}$ its area element. The derivation of this boundary functional is classical $[4,35]$ and leads to the following velocity vector:

$$
\mathbf{v}_{b}=[g(\mathbf{x}) \kappa-\nabla g(\mathbf{x}) \cdot \mathbf{N}] \mathbf{N},
$$

where $\mathbf{N}$ is the inward unit normal vector of $\Gamma$ and $\kappa$ its mean curvature. The idea is to use a local parametrization of $\Gamma$ to reduce (2.2) to a standard problem in the calculus of variations.

A region functional, $J_{r}$, may be expressed as an integral in a domain $\Omega$ of $\mathcal{U}$ of some function $f$ of some region features:

$$
J_{r}(\Omega)=\int_{\Omega} f(\mathbf{x}, \Omega) d \mathbf{x} .
$$


In that case, the computation of the velocity vector for (2.1) is not as easy. We propose comparing two main approaches. The first approach is based upon the idea of transforming all functionals into boundary functionals, thereby reducing (through a local parametrization of the boundary) the problem of minimization to a standard problem in the calculus of variations from which the computation of the Gâteaux derivatives follows. The second approach is based upon the use of shape derivation tools. In a sense it is more natural since it keeps the region representation.

Note that the scalar function $f$ in (2.3) is generally region-dependent. This is important since this dependency on the region must be taken into account when searching for a local minimum of the functional, as discussed in later sections.

Also note that we could have added a dependency of $g$ on $\partial \Omega$, i.e., write $g(\mathbf{x}, \partial \Omega)$ in (2.2), to keep the symmetry with the region functional. This is not necessary since the results in section 4.2, in particular Theorem 4.6, do in fact provide an answer for this case.

2.2. Examples of such optimization problems in image processing. An image is represented by its intensity $I(\mathbf{x})$ defined on some region of $\mathbb{R}^{n}$.

Active contours were originally introduced to search for minima of boundary functionals. In $[4,35]$, the function $g$ is a function of the magnitude of the image gradient through a strictly decreasing function $\varphi:\left[0,+\infty\left[\rightarrow \mathbb{R}^{+}\right.\right.$such that $\varphi(r) \rightarrow 0$ as $r \rightarrow+\infty$. Hence $g(\mathbf{x})=\varphi(|\nabla I(\mathbf{x})|)$. The minimization amounts to the minimization of the length of a curve in a Riemannian space. Local minima are obtained via the steepest descent method.

Region functionals have also been introduced. The region information is embedded in the function $f$ of (2.3). These functionals have been used for many applications such as moving objects detection [38, 40, 30, 32], image segmentation $[5,21,7,39,40,51]$, or classification $[52,44,45,41]$. For example, people have used statistical features such as the mean or the variance of a region $\Omega$ :

$$
\left\{\begin{aligned}
\mu_{\Omega} & =\frac{1}{|\Omega|} \int_{\Omega} I(\mathbf{x}) d \mathbf{x} \quad \text { with } \quad|\Omega|=\int_{\Omega} d \mathbf{x}, \\
\sigma_{\Omega}^{2} & =\frac{1}{|\Omega|} \int_{\Omega}\left(I(\mathbf{x})-\mu_{\Omega}\right)^{2} d \mathbf{x} .
\end{aligned}\right.
$$
tional

We use these two examples to motivate the introduction of a general region func-

$$
J_{r}(\Omega)=\int_{\Omega} f\left(\mathbf{x}, G_{1}(\Omega), G_{2}(\Omega), \ldots, G_{m}(\Omega)\right) d \mathbf{x},
$$

where the functionals $G_{i}$ are given by

$$
G_{i}(\Omega)=\int_{\Omega} H_{i}(\mathbf{x}, \Omega) d \mathbf{x}, \quad i=1 \ldots m .
$$

As shown in this equation, the function $H_{i}$ is itself region-dependent; more precisely,

$$
H_{i}(\mathbf{x}, \Omega) \stackrel{\text { def }}{=} H_{i}\left(\mathbf{x}, K_{i 1}(\Omega), K_{i 2}(\Omega), \ldots, K_{i l_{i}}(\Omega)\right),
$$

where

$$
K_{i j}(\Omega)=\int_{\Omega} L_{i j}(\mathbf{x}) d \mathbf{x}, \quad j=1 \ldots l_{i} \quad i=1 \ldots m
$$


Note that we have stopped the process at the second level but it could conceivably continue. We have chosen this special case of dependency because it often arises in applications, as shown in the next two sections. The various methods that we develop can be extended in a fairly straightforward fashion to more complicated situations if needed; see, for example, section 6 .

2.3. An example involving the mean. Let us choose

$$
f(\mathbf{x}, \Omega)=\varrho\left(I(\mathbf{x})-\mu_{\Omega}\right),
$$

where $\varrho: \mathbf{R} \rightarrow \mathbf{R}^{+}$is a positive function of class $C^{1} . f$ is region-dependent. This is an example where the process described in the previous section stops at the first level:

$$
J(\Omega)=\int_{\Omega} f(\mathbf{x}, \Omega) d \mathbf{x}=\int_{\Omega} \varrho\left(I(\mathbf{x})-\mu_{\Omega}\right) d \mathbf{x}=\int_{\Omega} \varrho\left(I(\mathbf{x})-\frac{G_{1}(\Omega)}{\left.G_{2}(\Omega)\right)}\right) d \mathbf{x},
$$

where

$$
\begin{aligned}
& G_{1}(\Omega)=\int_{\Omega} H_{1}(\mathbf{x}, \Omega) d \mathbf{x} \quad \text { with } \quad H_{1}(\mathbf{x}, \Omega)=I(\mathbf{x}), \\
& G_{2}(\Omega)=\int_{\Omega} H_{2}(\mathbf{x}, \Omega) d \mathbf{x} \quad \text { with } \quad H_{2}(\mathbf{x}, \Omega)=1 .
\end{aligned}
$$

In this case, the functions $H_{i}, i=1,2$, do not depend on the region $\Omega, l_{1}=l_{2}=0$, and $K_{i j}(\mathbf{x})=0$ for all $i, j$.

2.4. An example involving the variance. Let us take an example where we stop the process at the second level. Consider the case where the function $f$ is a function of the variance given by

$$
f(\mathbf{x}, \Omega)=\varrho\left(\sigma_{\Omega}^{2}\right) .
$$

$\varrho: \mathbf{R}^{+} \rightarrow \mathbf{R}^{+}$is of class $C^{1}$. We write

$$
J(\Omega)=\int_{\Omega} f(\mathbf{x}, \Omega) d \mathbf{x}=\int_{\Omega} \varrho\left(\sigma_{\Omega}^{2}\right) d \mathbf{x}=\int_{\Omega} \varrho\left(\frac{G_{1}(\Omega)}{\left.G_{2}(\Omega)\right)}\right) d \mathbf{x} .
$$

Therefore we have

$$
\begin{array}{ll}
G_{1}(\Omega) & =\int_{\Omega} H_{1}(\mathbf{x}, \Omega) d \mathbf{x}, \quad H_{1}(\mathbf{x}, \Omega)=\left(I(\mathbf{x})-\mu_{\Omega}\right)^{2}, \\
G_{2}(\Omega) & =\int_{\Omega} H_{2}(\mathbf{x}, \Omega) d \mathbf{x}, \quad H_{2}(\mathbf{x}, \Omega)=1
\end{array}
$$

with

$$
\begin{aligned}
& H_{1}(\mathbf{x}, \Omega)=\left(I(\mathbf{x})-\frac{K_{11}}{K_{12}}\right)^{2}, \quad l_{1}=2 \\
& H_{2}(\mathbf{x}, \Omega)=1, \quad l_{2}=0
\end{aligned}
$$

and finally

$$
\begin{aligned}
& K_{11}(\Omega)=\int_{\Omega} I(\mathbf{x}) d \mathbf{x}, \quad L_{11}(\mathbf{x})=I(\mathbf{x}) \\
& K_{12}(\Omega)=\int_{\Omega} d \mathbf{x}, \quad L_{12}(\mathbf{x})=1
\end{aligned}
$$


3. Expression of region functionals as boundary functionals and vice versa. In this section, we show that a region functional may always be expressed as a boundary functional and vice versa.

3.1. Tranformation of region functionals into boundary functionals. Consider the region functional (2.3); the next proposition shows that, under some reasonable assumptions on the function $f$, it can always be turned into a boundary functional (2.2).

Proposition 3.1. Let $\Omega$ be a bounded open set with regular boundary $\partial \Omega$. Let $f: \bar{\Omega} \rightarrow \mathbb{R}$ be a continuous function and $u$ be the unique solution of Poisson's equation:

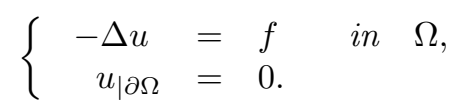

We have the following equality:

$$
\int_{\Omega} f(\mathbf{x}, \Omega) d \mathbf{x}=\int_{\partial \Omega} \nabla u \cdot \mathbf{N} d \mathbf{a}(\mathbf{x})
$$

where $\mathbf{N}$ is the inside pointing unit normal to $\partial \Omega$ and $d \mathbf{a}(\mathbf{x})$ its area element.

Proof. Because of our assumptions, Poisson's equation has a unique classical, i.e., $C^{2}$, solution in $\bar{\Omega}[2,25]$, and we have

$$
\int_{\Omega} f(\mathbf{x}, \Omega) d \mathbf{x}=-\int_{\Omega} \Delta u d \mathbf{x}=\int_{\partial \Omega} \nabla u \cdot \mathbf{N} d \mathbf{a}(\mathbf{x}),
$$

the last equality being a consequence of the Green-Riemann theorem.

A region functional can always be expressed as a boundary functional, via the solution of Poisson's equation with Dirichlet conditions.

3.2. Tranformation of boundary functionals into region functionals. The converse of Proposition 3.1 is also true. Let us consider the boundary functional (2.2).

Proposition 3.2. Let $\Omega$ be a bounded open set with regular boundary $\partial \Omega$. Let $u$ be the unique solution of Helmholtz's equation:

$$
\left\{\begin{aligned}
-\Delta u+u & =0 \quad \text { in } \quad \Omega, \\
\frac{\partial u}{\partial \mathbf{N}} \mid \partial \Omega & =-g .
\end{aligned}\right.
$$

Then we have the following equality:

$$
\int_{\partial \Omega} g(\mathbf{x}) d \mathbf{a}(\mathbf{x})=\int_{\Omega} u(\mathbf{x}, \Omega) d \mathbf{x}
$$

where $d \mathbf{a}(\mathbf{x})$ is the area element of $\partial \Omega$.

Proof. Because of our assumptions, Helmholtz's equation has a unique classical, i.e., $C^{2}$, solution in $\bar{\Omega}[42,13,14,15,16,17,18]$, and we have

$$
\int_{\Omega} u d \mathbf{x}=\int_{\Omega} \Delta u d \mathbf{x}=-\int_{\partial \Omega} \nabla u \cdot \mathbf{N} d \mathbf{a}(\mathbf{x}),
$$

the last equality being a consequence of the Green-Riemann theorem. Therefore

$$
\int_{\Omega} u d \mathbf{x}=-\int_{\partial \Omega} \frac{\partial u}{\partial \mathbf{N}} d \mathbf{a}(\mathbf{x})=\int_{\partial \Omega} g(\mathbf{x}) d \mathbf{a}(\mathbf{x}) .
$$

A boundary functional can always be expressed as a region functional, via the solution of Helmholtz's equation with Neumann boundary conditions. 
4. Computation of the evolution equation using a boundary approach. Originally, the derivation of region functionals has been performed by using the GreenRiemann theorem to transform region functionals into boundary functionals and then by computing the Euler-Lagrange equations. In this section, we recall the principles of the derivation and we explicitly take into account the case of region-dependent features when computing the Gâteaux derivative. Region functionals are transformed into boundary functionals by using Proposition 3.2. The region functional to minimize is $(2.3)$.

The computation of a velocity field for the evolution of the boundary in order to reach a minimum of the error criterion proceeds in three main steps:

1. Tranformation of the region functionals into boundary functionals.

2. Computation of the Gâteaux derivatives of the boundary functionals.

3. Construction of a velocity field for the evolution of the boundary.

The first step can always be performed as it has been proven in Proposition 3.1.

The computation of an optimal velocity field is carried out for region-independent features first, i.e., when the function $f$ does not depend on $\Omega$. We then consider the more general case where $f$ has some region dependency. We derive our results in the two-dimensional case; the generalization to any dimension is tedious but straightforward.

4.1. Region-independent features. In this part, we detail the three steps for region-independent features. We do it for two-dimensional images $(n=2)$ to keep notation simple, but the results hold in any dimension greater than 2 .

We parameterize $\partial \Omega$ through the $C^{2}$ function $\Gamma:[0,1] \rightarrow \mathbb{R}^{2}$ such that when $p$ varies from 0 to 1 we go once around $\partial \Omega$ counterclockwise. The unit tangent vector to $\partial \Omega$ is the vector $\Gamma^{\prime}(p) /\left|\Gamma^{\prime}(p)\right|$, where ' indicates the derivative with respect to the parameter $p$. The inside pointing normal $\mathbf{N}$ is the vector $\Gamma^{\prime} \perp(p) /\left|\Gamma^{\prime}(p)\right|$. The vector $\Gamma^{\prime} \perp$ is obtained by rotating $\Gamma^{\prime}$ by 90 degrees counterclockwise; hence if $\Gamma^{\prime}=\left[\Gamma_{1}^{\prime}, \Gamma_{2}^{\prime}\right]^{T}$, $\Gamma^{\prime} \perp=\left[-\Gamma_{2}^{\prime}, \Gamma_{1}^{\prime}\right]^{T}$.

4.1.1. Transformation of region functionals into boundary functionals. The following proposition is a straightforward consequence of Proposition 3.1

Proposition 4.1. If $f$ satisfies the hypotheses of Proposition 3.1, the functional (2.3),

$$
J_{r}(\Omega)=\int_{\Omega} f(\mathbf{x}) d \mathbf{x}
$$

is equal to

$$
\Phi(\Gamma)=\int_{0}^{1}\left(u_{x_{2}}(\Gamma(p)) \Gamma_{1}^{\prime}(p)-u_{x_{1}}(\Gamma(p)) \Gamma_{2}^{\prime}(p)\right) d p \stackrel{\text { def }}{=} \int_{0}^{1} \varphi\left(\Gamma(p), \Gamma^{\prime}(p)\right) d p,
$$

where $\Gamma=\partial \Omega$ and $u$ is the unique classical solution of

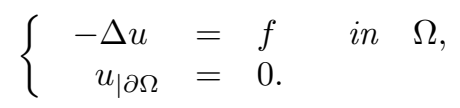

Therefore minimizing (2.3) with respect to $\Omega$ is equivalent to minimizing (4.1) with respect to $\Gamma$.

Proof. According to Proposition 3.1, we have

$$
\int_{\Omega} f(\mathbf{x}) d \mathbf{x}=-\int_{\Omega} \Delta u d \mathbf{x}=\int_{\partial \Omega} \nabla u \cdot \mathbf{N} d \mathbf{a}(\mathbf{x}),
$$

and since $d \mathbf{a}(\mathbf{x})=\left|\Gamma^{\prime}(p)\right| d p$, the result follows. 
4.1.2. Computation of the Gâteaux derivative. According to Proposition 4.1, minimizing (2.3) with respect to $\Omega$ is equivalent to minimizing (4.1) with respect to $\Gamma$. Thus, we have to compute the Gâteaux derivative of the functional $\Phi$.

Theorem 4.2. The Gâteaux derivative in the direction $\gamma$ of the functional $\Phi$ is

$$
\left\langle\Phi^{\prime}(\Gamma), \gamma\right\rangle=-\int_{0}^{1} f(\Gamma(p))\left(\Gamma^{\prime \perp}(p) \cdot \gamma(p)\right) d p .
$$

Proof. Let $\gamma:[0,1] \rightarrow \mathbf{R}^{2}$ be a $C^{2}$ parametrization of an arbitrary closed curve. The Gâteaux derivative of $\Phi(\Gamma)$ in the direction $\gamma$ noted $\left.\left\langle\Phi^{\prime}(\Gamma), \gamma\right\rangle\right\rangle$ is defined by

$$
\left\langle\Phi^{\prime}(\Gamma), \gamma\right\rangle=\lim _{\tau \rightarrow 0} \frac{\Phi(\Gamma+\tau \gamma)-\Phi(\Gamma)}{\tau} .
$$

We have

$$
\lim _{\tau \rightarrow 0} \frac{\Phi(\Gamma+\tau \gamma)-\Phi(\Gamma)}{\tau}=\int_{0}^{1}\left(\varphi_{\Gamma}\left(\Gamma(p), \Gamma^{\prime}(p)\right) \gamma(p)+\varphi_{\Gamma^{\prime}}\left(\Gamma(p), \Gamma^{\prime}(p)\right) \gamma^{\prime}(p)\right) d p,
$$

where $\varphi_{\Gamma}=\frac{\partial \varphi}{\partial \Gamma}\left(\Gamma, \Gamma^{\prime}\right)$. Integrating by parts, we obtain the following expression for the Gâteaux derivative:

$$
\left\langle\Phi^{\prime}(\Gamma), \gamma\right\rangle=\int_{0}^{1}\left(\varphi_{\Gamma}\left(\Gamma(p), \Gamma^{\prime}(p)\right)-\frac{d}{d p} \varphi_{\Gamma^{\prime}}\left(\Gamma(p), \Gamma^{\prime}(p)\right)\right) \cdot \gamma(p) d p .
$$

We then explicitly compute the derivative of $\varphi$ with respect to $\Gamma$ using (4.1),

$$
\varphi_{\Gamma}=\nabla u_{x_{2}}(\Gamma(p)) \Gamma_{1}^{\prime}(p)-\nabla u_{x_{1}}(\Gamma(p)) \Gamma_{2}^{\prime}(p),
$$

and with respect to $\Gamma^{\prime}$,

$$
\varphi_{\Gamma^{\prime}}=\left[u_{x_{2}},-u_{x_{1}}\right]^{T}
$$

Therefore

$$
\frac{d}{d p} \varphi_{\Gamma^{\prime}}=\left[\nabla u_{x_{2}} \cdot \Gamma^{\prime},-\nabla u_{x_{1}} \cdot \Gamma^{\prime}\right]^{T} .
$$

Putting everything together we obtain

$$
\varphi_{\Gamma}-\frac{d}{d p} \varphi_{\Gamma^{\prime}}=\Delta u \Gamma^{\prime} \perp=-f \Gamma^{\prime} \perp
$$

thanks to Proposition 4.1.

The Euler-Lagrange equations associated with the Gâteaux derivative are thus given by

$$
\varphi_{\Gamma}-\frac{d}{d p} \varphi_{\Gamma^{\prime}}=-f(\Gamma(p)) \Gamma^{\prime \perp} .
$$

An interesting point to note is that the intermediary function $u$ disappears.

4.1.3. Construction of an optimal velocity vector for the evolution of an active contour. In order to find a local extremum of the criterion (4.1), we evolve a curve using the steepest descent method, starting from an initial curve defined by the user. Thus, we obtain the following evolution equation:

$$
\frac{\partial \Gamma}{\partial \tau}=f(\Gamma) \mathbf{N} \quad \text { with } \Gamma(\tau=0)=\Gamma_{0} .
$$

This is the classical result $[52,38,40,51]$ when $f$ has no region dependency. Let us now consider the more general case where the function $f$ has some region dependency. 
4.2. General case. Let us now derive the evolution equation in the general case. As in the previous case, we follow our three steps.

4.2.1. Transformation of the region functional into a boundary functional. In the following, to simplify the proofs and the notations, we take $m=1$ and $l_{1}=1$ and drop the indexes. The equations for $m>1$ and $l_{i} \geq 1$ are then given without proof.

Because of the form of (2.4)-(2.7), we have to go through three levels of transformations. We start with the first level and the following proposition.

Proposition 4.3. If $L$ satisfies the assumptions of Proposition 3.1, the functional

$$
K(\Omega)=\int_{\Omega} L(\mathbf{x}) d \mathbf{x}
$$

is equal to

$$
\begin{aligned}
\Theta(\Gamma) & =\int_{0}^{1}\left(u_{x_{2}}(\Gamma(p), L(\Gamma)) \Gamma_{1}^{\prime}(p)-u_{x_{1}}(\Gamma(p), L(\Gamma)) \Gamma_{2}^{\prime}(p)\right) d p \\
& \stackrel{\text { def }}{=} \int_{0}^{1} \theta\left(\Gamma(p), \Gamma^{\prime}(p)\right) d p
\end{aligned}
$$

where $\Gamma=\partial \Omega$ and $u$ is the unique classical solution of

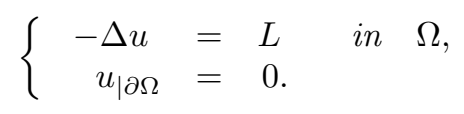

Proof. The proof is identical to that of Proposition 4.1.

In the same manner, for the second level, we have the following proposition.

Proposition 4.4. If $H$ satisfies the assumptions of Proposition 3.1, the functional

$$
G(\Omega)=\int_{\Omega} H(\mathbf{x}, K(\Omega)) d \mathbf{x}
$$

with $K(\Omega)=\int_{\Omega} L(\mathbf{x}) d \mathbf{x}$ is equal to

$$
\begin{gathered}
\Psi(\Gamma)=\int_{0}^{1}\left(v_{x_{2}}(\Gamma(p), \Theta(\Gamma)) \Gamma_{1}^{\prime}(p)-v_{x_{1}}(\Gamma(p), \Theta(\Gamma)) \Gamma_{2}^{\prime}(p)\right) d p \\
\stackrel{\text { def }}{=} \int_{0}^{1} \psi\left(\Gamma(p), \Gamma^{\prime}(p), \Theta(\Gamma)\right) d p
\end{gathered}
$$

where $\Gamma=\partial \Omega$ and $v$ is the unique classical solution of

$$
\left\{\begin{aligned}
-\Delta v & =H \quad \text { in } \quad \Omega \\
v_{\mid \partial \Omega} & =0 .
\end{aligned}\right.
$$

$\Theta$ is given by Proposition 4.3.

Proof. The proof is identical to that of Proposition 4.1.

We finally reach the third and last level with the following proposition.

Proposition 4.5. If $f$ satisfies the assumptions of Proposition 3.1, the functional

$$
J(\Omega)=\int_{\Omega} f(\mathbf{x}, G(\Omega)) d \mathbf{x},
$$


with $G(\Omega)=\int_{\Omega} H(\mathbf{x}, K(\Omega)) d \mathbf{x}$ and $K(\Omega)=\int_{\Omega} L(\mathbf{x}) d \mathbf{x}$, is equal to

$$
\begin{aligned}
\Phi(\Gamma) & =\int_{0}^{1}\left(w_{x_{2}}(\Gamma(p), \Psi(\Gamma)) \Gamma_{1}^{\prime}(p)-w_{x_{1}}(\Gamma(p), \Psi(\Gamma)) \Gamma_{2}^{\prime}(p)\right) d p \\
& \stackrel{\text { def }}{=} \int_{0}^{1} \varphi\left(\Gamma(p), \Gamma^{\prime}(p), \Psi(\Gamma)\right) d p,
\end{aligned}
$$

where $\Gamma=\partial \Omega$ and $u$ is the unique classical solution of

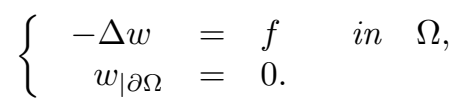

$\Psi(\Gamma)$ is given by Proposition 4.4. Therefore minimizing (4.3) with respect to $\Omega$ is equivalent to minimizing (4.4) with respect to $\Gamma$.

Proof. The proof is identical to that of Proposition 4.1.

4.2.2. Computation of the Gâteaux derivative. According to Proposition 4.5, minimizing (4.3) with respect to $\Omega$ is equivalent to minimizing (4.4) with respect to $\Gamma$. Thus we compute the Gâteaux derivative of $\Phi$ given by (4.4).

TheOREM 4.6. The Gâteaux derivative in the direction $\gamma$ of the functional $\Phi$ defined in (4.4) is

$$
\begin{gathered}
\left\langle\Phi^{\prime}(\Gamma), \gamma\right\rangle=-\int_{0}^{1}[f(\Gamma(p), \Psi(\Gamma))+A H(\Gamma(p), \Theta(\Gamma)) \\
+A B L(\Gamma(p))] q(p) d p
\end{gathered}
$$

where

$$
A=\int_{\Omega} f_{G}(x, G(\Omega)) d \mathbf{x} \quad \text { and } \quad B=\int_{\Omega} H_{K}(x, K(\Omega)) d \mathbf{x}
$$

with $f_{G}=\frac{\partial f}{\partial G}$, and $q(p)=\left(\Gamma^{\prime} \perp(p) \cdot \gamma(p)\right)$.

Proof. The Gâteaux derivative of $\Phi(\Gamma)$ in the direction $\gamma$ denoted $\left\langle\Phi^{\prime}(\Gamma), \gamma\right\rangle$ is given by

$$
\left\langle\Phi^{\prime}(\Gamma), \gamma\right\rangle=\lim _{\tau \rightarrow 0} \frac{\Phi(\Gamma+\tau \gamma)-\Phi(\Gamma)}{\tau}
$$

We have

$$
\begin{aligned}
\lim _{\tau \rightarrow 0} & \frac{\Phi+\tau \gamma)-\Phi(\Gamma)}{\tau} \\
= & \int_{0}^{1}\left(\varphi_{\Gamma}\left(\Gamma(p), \Gamma^{\prime}(p), \Psi(\Gamma)\right) \gamma(p)+\varphi_{\Gamma^{\prime}}\left(\Gamma(p), \Gamma^{\prime}(p), \Psi(\Gamma)\right) \gamma^{\prime}(p)\right) d p \\
& +\int_{0}^{1} \varphi_{\Psi}\left(\Gamma(p), \Gamma^{\prime}(p), \Psi(\Gamma)\right)\left\langle\Psi^{\prime}(\Gamma), \gamma\right\rangle d p,
\end{aligned}
$$

where $\varphi_{\Psi}=\frac{\partial \varphi}{\partial \Psi}\left(\Gamma, \Gamma^{\prime}, \Psi\right)$. Integrating by parts, we obtain

$$
\begin{aligned}
\left\langle\Phi^{\prime}(\Gamma), \gamma\right\rangle & =\int_{0}^{1}\left[\varphi_{\Gamma}-\frac{d}{d p} \varphi_{\Gamma^{\prime}}\right] \gamma(p) d p \\
& +\int_{0}^{1} \varphi_{\Psi}\left(\Gamma(p), \Gamma^{\prime}(p), \Psi(\Gamma)\right)\left\langle\Psi^{\prime}(\Gamma), \gamma\right\rangle d p .
\end{aligned}
$$


According to Theorem 4.2, we obtain $\varphi_{\Gamma}-\frac{d}{d p} \varphi_{\Gamma^{\prime}}=-f \Gamma^{\prime} \perp$. The Gâteaux derivative of $\Psi(\Gamma)$ in the direction $\gamma$ is computed in the same manner and we find

$$
\begin{aligned}
\left\langle\Psi^{\prime}(\Gamma), \gamma\right\rangle= & -\int_{0}^{1} H(\Gamma(p), \Theta(\Gamma)) q(p) d p \\
& +\int_{0}^{1} \psi_{\Theta}\left(\Gamma(p), \Gamma^{\prime}(p), \Theta(\Gamma)\right)\left\langle\Theta^{\prime}(\Gamma), \gamma\right\rangle d p .
\end{aligned}
$$

According to Theorem 4.2, the Gâteaux derivative of $\Theta(\Gamma)$ in the direction $\gamma$ is given by:

$$
\left\langle\Theta^{\prime}(\Gamma), \gamma\right\rangle=-\int_{0}^{1} L(\Gamma(p)) q(p) d p
$$

Putting all terms together in (4.5), we find the following expression for the derivative:

$$
\begin{aligned}
\left\langle\Phi^{\prime}(\Gamma), \gamma\right\rangle= & -\int_{0}^{1} f(\Gamma(p), \Psi(\Gamma)) q(p) d p \\
& -\int_{0}^{1} \varphi_{\Psi}\left(\Gamma(p), \Gamma^{\prime}(p), \Psi(\Gamma)\right) d p \int_{0}^{1} H(\Gamma(p), \Theta(\Gamma)) q(p) d p \\
- & \int_{0}^{1} \varphi_{\Psi}\left(\Gamma(p), \Gamma^{\prime}(p), \Psi(\Gamma)\right) d p \int_{0}^{1} \psi_{\Theta}\left(\Gamma(p), \Gamma^{\prime}(p), \Theta(\Gamma)\right) d p \int_{0}^{1} L(\Gamma(p)) q(p) d p .
\end{aligned}
$$

Using Propositions 4.4 and 4.5, we find that

$$
\int_{0}^{1} \varphi_{\Psi}\left(\Gamma(p), \Gamma^{\prime}(p), \Psi(\Gamma)\right) d p=\int_{\Omega} f_{G}(\mathbf{x}, G(\Omega)) d \mathbf{x} \stackrel{\text { def }}{=} A .
$$

Similarly, using Propositions 4.3 and 4.4, we obtain

$$
\int_{0}^{1} \psi_{\Theta}\left(\Gamma(p), \Gamma^{\prime}(p), \Theta(\Gamma)\right) d p=\int_{\Omega} H_{K}(\mathbf{x}, K(\Omega)) d \mathbf{x} \stackrel{\text { def }}{=} B
$$

The equation of the derivative is obtained:

$$
\left\langle\Phi^{\prime}(\Gamma), \gamma\right\rangle=-\int_{0}^{1}[f(\Gamma(p), \Psi(\Gamma))+A H(\Gamma(p), \Theta(\Gamma))+A B L(\Gamma(p))] q(p) d p .
$$

The Euler-Lagrange equations associated with the Gâteaux derivative are given by

$$
-[f(\Gamma(p), \Psi(\Gamma))+A H(\Gamma(p), \Theta(\Gamma))+A B L(\Gamma(p))] \Gamma^{\prime} \perp=0 .
$$

Note again that the intermediate functions $u, v$, and $w$ do not appear in this expression.

We can now state the general theorem for $m>1$ and $l_{i} \geq 1$.

TheOrem 4.7. The Gâteaux derivative in the direction $\gamma$ of the functional $J$ 
defined in (2.4) is

$$
\begin{aligned}
\left\langle\Phi^{\prime}(\Gamma), \gamma\right\rangle=-\int_{0}^{1} & \left(f\left(\Gamma(p), G_{1}(\Gamma), \ldots, G_{m}(\Gamma)\right)\right. \\
& +\sum_{i=1}^{m} A_{i} H_{i}\left(\Gamma(p), K_{i 1}(\Gamma), \ldots, K_{i l_{i}}(\Gamma)\right) \\
& \left.+\sum_{i=1}^{m} A_{i}\left(\sum_{j=1}^{l_{i}} B_{i j} L_{i j}(\Gamma(p))\right)\right)\left(\Gamma^{\prime} \perp(p) \cdot \gamma(p)\right) d p
\end{aligned}
$$

where

$$
\begin{aligned}
A_{i} & =\int_{\Omega} f_{G_{i}}\left(x, G_{1}(\Omega), \ldots, G_{m}(\Omega)\right) d \mathbf{x}, \quad i=1 \ldots m \\
\text { and } \quad B_{i j} & =\int_{\Omega} H_{i K_{i j}}\left(x, K_{i 1}(\Omega), \ldots, K_{i l_{i}}(\Omega)\right) d \mathbf{x}, \quad i=1 \ldots m, \quad j=1 \ldots l_{i} .
\end{aligned}
$$

4.2.3. Construction of an optimal velocity vector for the evolution of an active contour. In the general case, according to Theorem 4.7 the steepest gradient descent method yields the following evolution equation for the active contour:

$$
\frac{\partial \Gamma}{\partial \tau}=\left[f(\Gamma)+\sum_{i=1}^{m} A_{i} H_{i}(\Gamma)+\sum_{i=1}^{m} A_{i}\left(\sum_{j=1}^{l_{i}} B_{i j} L_{i j}(\Gamma)\right)\right] \mathbf{N}
$$

with $\Gamma(\tau=0)=\Gamma_{0}$. Compared with (4.2), some additional terms appear that come from the region dependency of the descriptors.

5. Computation of the derivative using shape derivation tools, or "how to keep a region formulation." In the previous part, region functionals were first transformed into boundary functionals for the computation of the derivative. This step is neither natural nor straightforward. Therefore, we propose another solution based on shape derivation tools $[49,22]$. The region formulation is maintained for the computation and this provides a suitable way of obtaining the derivative of the criterion and the evolution equation of an active contour.

We perform three main steps:

1. Introduction of a dynamic scheme. Since the set of all image regions is not a vector space, it is difficult to compute the derivative of the criterion with respect to the domain $\Omega$. To circumvent this problem, we apply a family of transformations $T_{\tau}$, indexed by a real parameter $\tau \geq 0$, to $\Omega$, and we denote $\Omega(\tau)=T_{\tau}(\Omega)$. The region functional becomes a function of $\tau, J(\tau) \stackrel{\text { def }}{=}$ $J(\Omega(\tau))$.

2. Derivation of the criterion based on shape derivation principles. The error criterion $J(\tau)$ is then derived with respect to $\tau$ using shape derivation principles.

3. Computation of the evolution equation from the derivative. From the derivative, we deduce the velocity field of the active contour that will make it evolve towards a local minimum of the error criterion. 
5.1. Introduction of transformations. As it has already been pointed out, the optimization of the region functional (2.3) is difficult since the set of regular domains (regular open bounded sets) $\mathcal{U}$ of $\mathbb{R}^{n}$ does not have the structure of a vector space. Variations of a domain must then be defined in some way. Let us consider a reference domain $\Omega \in \mathcal{U}$ and the set $\hat{\mathcal{A}}$ of applications $T: \Omega \rightarrow \mathbb{R}^{n}$, which are at least as regular as homeomorphisms (i.e., one to one with $T$ and $T^{-1}$ continuous). We define

$$
\hat{\mathcal{A}}=\left\{T \text { one to one, } T, T^{-1} \in W^{1, \infty}\left(\Omega, \mathbb{R}^{n}\right)\right\},
$$

where

$$
\begin{aligned}
W^{1, \infty}\left(\Omega, \mathbb{R}^{n}\right)=\left\{T: \Omega \rightarrow \mathbb{R}^{n} \text { such that } T \in L^{\infty}\left(\Omega, \mathbb{R}^{n}\right)\right. \text { and } \\
\left.\qquad \partial_{i} T \in L^{\infty}\left(\Omega, \mathbb{R}^{n}\right), i=1, \ldots, n\right\} .
\end{aligned}
$$

Given a shape function $F: \mathcal{U} \rightarrow \mathbf{R}^{+}$for $T \in \hat{\mathcal{A}}$, let us define $\hat{F}(T)=F(T(\Omega))$. The key point is that $W^{1, \infty}\left(\Omega, \mathbb{R}^{n}\right)$ is a Banach space. This allows us to define the notion of derivative with respect to the domain $\Omega$ as follows.

Definition 5.1. F is Gâteaux differentiable with respect to $\Omega$ if and only if $\hat{F}$ is Gâteaux differentiable with respect to T.

In order to compute Gâteaux derivatives with respect to $T$ we introduce a family of deformation $(T(\tau))_{\tau \geq 0}$ such that $T(\tau) \in \hat{\mathcal{A}}$ for $\tau \geq 0, T(0)=\mathrm{Id}$, and $T(.) \in$ $C^{1}\left([0, A] ; W^{1, \infty}\left(\Omega, \mathbb{R}^{n}\right)\right), A>0$. From a practical point of view, there are many ways to construct such a family. The most famous one is the Hadamard deformation [27].

For a point $\mathrm{x} \in \Omega$, we denote

$$
\begin{gathered}
\mathbf{x}(\tau)=T(\tau, \mathbf{x}) \text { with } \quad T(0, \mathbf{x})=\mathbf{x}, \\
\Omega(\tau)=T(\tau, \Omega) \text { with } T(0, \Omega)=\Omega .
\end{gathered}
$$

Let us now define the velocity vector field $\mathbf{V}$ corresponding to $T(\tau)$ as

$$
\mathbf{V}(\tau, \mathbf{x})=\frac{\partial T}{\partial \tau}(\tau, \mathbf{x}) \quad \forall \mathbf{x} \in \Omega \quad \forall \tau \geq 0 .
$$

5.2. Computation of the derivative using shape derivation tools. We now introduce three main definitions.

Definition 5.2. The Gâteaux derivative of $J(\Omega)=\int_{\Omega} f(\mathbf{x}, \Omega) d \mathbf{x}$ in the direction of $\mathbf{V}$, denoted $\left\langle J^{\prime}(\Omega), \mathbf{V}\right\rangle$, is equal to

$$
\left\langle J^{\prime}(\Omega), \mathbf{V}\right\rangle=\lim _{\tau \rightarrow 0} \frac{J(\Omega(\tau))-J(\Omega)}{\tau} .
$$

Definition 5.3. The material derivative of $f(\mathbf{x}, \Omega)$, denoted $f_{m}(\mathbf{x}, \Omega, \mathbf{V})$, is equal to

$$
f_{m}(\mathbf{x}, \Omega, \mathbf{V})=\lim _{\tau \rightarrow 0} \frac{f(\mathbf{x}(\tau), \Omega(\tau))-f(\mathbf{x}, \Omega)}{\tau} .
$$

Definition 5.4. The shape derivative of $f(\mathbf{x}, \Omega)$, denoted $f_{s}(\mathbf{x}, \Omega, \boldsymbol{V})$, is equal to

$$
f_{s}(\mathbf{x}, \Omega, \mathbf{V})=\lim _{\tau \rightarrow 0} \frac{f(\mathbf{x}, \Omega(\tau))-f(\mathbf{x}, \Omega)}{\tau} .
$$


5.2.1. Relation between the Gâteaux derivative and the shape derivative. The following theorem gives a relation between the Gâteaux derivative and the shape derivative for the region functional (2.3). The proof can be found in [49, 22], we provide an elementary one here for completeness.

TheOREM 5.5. The Gâteaux derivative of the functional $J(\Omega)=\int_{\Omega} f(\mathbf{x}, \Omega) d \mathbf{x}$ in the direction of $\mathbf{V}$ is the following:

$$
\left\langle J^{\prime}(\Omega), \mathbf{V}\right\rangle=\int_{\Omega} f_{s}(\mathbf{x}, \Omega, \mathbf{V}) d \mathbf{x}-\int_{\partial \Omega} f(\mathbf{x}, \Omega)(\mathbf{V}(\mathbf{x}) \cdot \mathbf{N}(\mathbf{x})) d \mathbf{a}(\mathbf{x}),
$$

where $\boldsymbol{N}$ is the unit inward normal to $\partial \Omega$ and da its area element.

Proof. As far as the computation of the derivative is concerned, only small deformations are relevant, and we thus consider a first order Taylor expansion of the transformation:

$$
\begin{aligned}
T(\tau, \mathbf{x}) & =T(0, \mathbf{x})+\tau \frac{\partial T}{\partial \tau}(0, \mathbf{x}) \\
& =\mathbf{x}+\tau \mathbf{V}(\mathbf{x})
\end{aligned}
$$

where $\mathbf{V}(\mathbf{x})=\frac{\partial T}{\partial \tau}(0, \mathbf{x})$.

We obtain the following expressions for the material and the shape derivatives:

$$
\begin{aligned}
f_{m}(\mathbf{x}, \Omega, \mathbf{V}) & =\lim _{\tau \rightarrow 0} \frac{f(\mathbf{x}+\tau \mathbf{V}(\mathbf{x}), \Omega+\tau \mathbf{V})-f(\mathbf{x}, \Omega)}{\tau}, \\
f_{s}(\mathbf{x}, \Omega, \mathbf{V}) & =\lim _{\tau \rightarrow 0} \frac{f(\mathbf{x}, \Omega+\tau \mathbf{V})-f(\mathbf{x}, \Omega)}{\tau} .
\end{aligned}
$$

If we assume that $\lim _{\tau \rightarrow 0} \nabla f(\mathbf{x}, \Omega+\tau \mathbf{V})=\nabla f(\mathbf{x}, \Omega)$, we can write

$$
f_{m}(\mathbf{x}, \Omega, \mathbf{V})=f_{s}(\mathbf{x}, \Omega, \mathbf{V})+\nabla f(\mathbf{x}, \Omega) \cdot \mathbf{V}(\mathbf{x}) .
$$

We are now ready for the computation of the Gâteaux derivative of $J(\Omega)$ in the direction of $\mathbf{V}$. We have

$$
\frac{J(\Omega(\tau))-J(\Omega)}{\tau}=\frac{1}{\tau}\left[\int_{\Omega(\tau)} f(\mathbf{x}, \Omega(\tau)) d \mathbf{x}-\int_{\Omega} f(\mathbf{x}, \Omega) d \mathbf{x}\right] .
$$

In the first integral, we make the change of variable $\mathbf{x} \rightarrow \mathbf{x}+\tau \mathbf{V}(\mathbf{x})$ which yields

$$
\int_{\Omega(\tau)} f(\mathbf{x}, \Omega(\tau)) d \mathbf{x}=\int_{\Omega} f(\mathbf{x}+\tau \mathbf{V}(\mathbf{x}), \Omega+\tau \mathbf{V})\left|\operatorname{det} J_{\tau}(\mathbf{x})\right| d \mathbf{x},
$$

where $J_{\tau}(\mathbf{x})$ is the Jacobian matrix,

$$
J_{\tau}(\mathbf{x})=\left(\begin{array}{ccc}
1+\tau \frac{\partial V_{1}}{\partial x_{1}} & \cdots & \tau \frac{\partial V_{1}}{\partial x_{n}} \\
\vdots & \vdots & \vdots \\
\tau \frac{\partial V_{n}}{\partial x_{1}} & \cdots & 1+\tau \frac{\partial V_{n}}{\partial x_{n}}
\end{array}\right)
$$

$\mathbf{V}(\mathbf{x})=\left[V_{1}(\mathbf{x}), \ldots, V_{n}(\mathbf{x})\right]^{T}$, and $\mathbf{x}=\left[x_{1}, \ldots, x_{n}\right]^{T}$. It follows that

$$
\operatorname{det} J_{\tau}(\mathbf{x})=1+\tau \operatorname{div}(\mathbf{V}(\mathbf{x}))+o(\tau) .
$$


This shows that if $\tau$ is small enough, $\operatorname{det} J_{\tau}(\mathbf{x})>0$ and

$$
\lim _{\tau \rightarrow 0} \frac{\operatorname{det} J_{\tau}(\mathbf{x})-1}{\tau}=\operatorname{div}(\mathbf{V}(\mathbf{x})) .
$$

Equation (5.2) can now be rewritten as

$$
\begin{aligned}
\frac{J(\Omega(\tau))-J(\Omega)}{\tau}= & \int_{\Omega} \frac{f(\mathbf{x}+\tau \mathbf{V}(\mathbf{x}), \Omega+\tau \mathbf{V})-f(\mathbf{x}, \Omega)}{\tau} \operatorname{det}\left(J_{\tau}(\mathbf{x})\right) d \mathbf{x} \\
& -\int_{\Omega} f(\mathbf{x}, \Omega) \frac{\operatorname{det}\left(J_{\tau}(\mathbf{x})\right)-1}{\tau} d \mathbf{x} \stackrel{\text { def }}{=} I_{1}-I_{2} .
\end{aligned}
$$

If $\tau$ goes to 0 , using (5.1) and Definitions 5.3 and 5.4, we get

$$
\begin{aligned}
\lim _{\tau \rightarrow 0} I_{1} & =\int_{\Omega} f_{m}(\mathbf{x}, \Omega, \mathbf{V}) d \mathbf{x} \\
& =\int_{\Omega} f_{s}(\mathbf{x}, \Omega, \mathbf{V}) d \mathbf{x}+\int_{\Omega} \nabla f(\mathbf{x}, \Omega) \cdot \mathbf{V}(\mathbf{x}) d \mathbf{x}, \\
\lim _{\tau \rightarrow 0} I_{2} & =\int_{\Omega} f(\mathbf{x}, \Omega) \operatorname{div}(\mathbf{V}(\mathbf{x})) d \mathbf{x} .
\end{aligned}
$$

We find that the Gâteaux derivative is given by

$$
\begin{aligned}
\left\langle J^{\prime}(\Omega), \mathbf{V}\right\rangle & =\int_{\Omega} f_{s}(\mathbf{x}, \Omega, \mathbf{V}) d \mathbf{x}+\int_{\Omega}(\nabla f(\mathbf{x}, \Omega) \cdot \mathbf{V}(\mathbf{x})+f(\mathbf{x}, \Omega) \operatorname{div}(\mathbf{V}(\mathbf{x}))) d \mathbf{x} \\
& =\int_{\Omega} f_{s}(\mathbf{x}, \Omega, \mathbf{V}) d \mathbf{x}+\int_{\Omega} \operatorname{div}(f(\mathbf{x}, \Omega) \mathbf{V}(\mathbf{x})) d \mathbf{x} .
\end{aligned}
$$

Applying the Green-Riemann theorem in (5.3), we finally obtain

$$
\left\langle J^{\prime}(\Omega), \mathbf{V}\right\rangle=\int_{\Omega} f_{s}(\mathbf{x}, \Omega, \mathbf{V}) d \mathbf{x}-\int_{\partial \Omega} f(\mathbf{x}, \Omega)(\mathbf{V}(\mathbf{x}) \cdot \mathbf{N}(\mathbf{x})) d \mathbf{a}(\mathbf{x}),
$$

where $\mathbf{N}$ is the unit inward normal to $\partial \Omega$.

Note that Theorem 5.5 provides a necessary condition for a domain $\hat{\Omega}$ to be an extremum of $J(\Omega)$ :

$$
\int_{\hat{\Omega}} f_{s}(\mathbf{x}, \hat{\Omega}, \mathbf{V}) d \mathbf{x}-\int_{\partial \hat{\Omega}} f(\mathbf{x}, \hat{\Omega})(\mathbf{V}(\mathbf{x}) \cdot \mathbf{N}(\mathbf{x})) d \mathbf{a}(\mathbf{x})=0 \quad \forall \mathbf{V} .
$$

5.3. Construction of the velocity vector of the active contour from the Gâteaux derivative. We now make good use of the previous tools to derive the velocity vector of the active contour for the same functionals as those which were considered in section 5 . As expected we find the same results but in a way which, we feel, is more natural, since we do not have to turn a region integral into a boundary one, and simpler. The evolving region boundary $\partial \Omega$, denoted $\Gamma$, is modeled as an active contour: the user defines an initial curve $\Gamma_{0}=\partial \Omega_{0}$ that evolves towards a local minimum of the region functional (2.3) according to a PDE that we will now derive.

5.3.1. Region-independent features. We first consider the simple case where the function $f$ does not depend on $\Omega$, i.e., $f=f(\mathbf{x})$. In that case, the shape derivative $f_{s}$ is equal to zero and the Gâteaux derivative of $J$ is simply (Theorem 5.5)

$$
\left\langle J^{\prime}(\Omega), \mathbf{V}\right\rangle=-\int_{\partial \Omega} f(\mathbf{x})(\mathbf{V}(\mathbf{x}) \cdot \mathbf{N}(\mathbf{x})) d \mathbf{a}(\mathbf{x}) .
$$


This leads to the following evolution equation for region-independent descriptors:

$$
\frac{\partial \Gamma}{\partial \tau}=f(\Gamma) \mathbf{N}
$$

with $\Gamma(\tau=0)=\Gamma_{0}$.

We notice that, as expected, the evolution equation is the same as (4.2) in section 4 .

5.3.2. General case. Let us now tackle the same general case as in section 4.2 , using the functional defined by (2.4)-(2.7). We similarly restrict the computation of the Gâteaux derivative of $J$ to the case $m=1$ and $l_{i}=1$, state the result for $m>1$ and $l_{i} \geq 1$, and drop the indexes.

TheOREm 5.6. The Gateaux derivative in the direction of $\mathbf{V}$ of the functional $J$ defined in (4.3) is

$$
\left\langle J^{\prime}(\Omega), \mathbf{V}\right\rangle=-\int_{\Gamma}(A B L(\mathbf{x})+A H(\mathbf{x}, K(\Omega))+f(\mathbf{x}, \Omega))(\mathbf{V}(\mathbf{x}) \cdot \mathbf{N}(\mathbf{x})) d \mathbf{a}(\mathbf{x}),
$$

where

$$
A=\int_{\Omega} f_{G}(x, G(\Omega)) d \mathbf{x} \quad \text { and } \quad B=\int_{\Omega} H_{K}(x, K(\Omega)) d \mathbf{x} .
$$

Proof. According to Theorem 5.5, we have

$$
\left\langle J^{\prime}(\Omega), \mathbf{V}\right\rangle=\int_{\Omega} f_{s} d \mathbf{x}-\int_{\Gamma} f(\mathbf{V} \cdot \mathbf{N}) d \mathbf{a}(\mathbf{x}) .
$$

Let us first compute the shape derivative of $f$. From the chain rule we get

$$
f_{s}(\mathbf{x}, \Omega, \mathbf{V})=f_{G}(\mathbf{x}, G)\left\langle G^{\prime}(\Omega), \mathbf{V}\right\rangle,
$$

where $f_{G}$ denotes the partial derivative of the function $f$ with respect to its second argument.

Next we compute the Gâteaux derivative of $G$ in the direction of $\mathbf{V}$. We again apply Theorem 5.5, and we get

$$
\left\langle G^{\prime}(\Omega), \mathbf{V}\right\rangle=\int_{\Omega} H_{s} d \mathbf{x}-\int_{\Gamma} H(\mathbf{V} \cdot \mathbf{N}) d \mathbf{a}(\mathbf{x}) .
$$

Plugging this into (5.4), we obtain

$$
\int_{\Omega} f_{s} d \mathbf{x}=A\left(\int_{\Omega} H_{s} d \mathbf{x}-\int_{\Gamma} H(\mathbf{V} \cdot \mathbf{N}) d \mathbf{a}(\mathbf{x})\right),
$$

where

$$
A=\int_{\Omega} f_{G}(\mathbf{x}, G(\Omega)) d \mathbf{x}
$$

We also compute the shape derivative of $H$ through Theorem 5.5:

$$
H_{s}(\mathbf{x}, \Omega, \mathbf{V})=H_{K}(\mathbf{x}, K)\left\langle K^{\prime}(\Omega), \mathbf{V}\right\rangle .
$$


We continue with the Gâteaux derivative of $K$ in the direction of $\mathbf{V}$ :

$$
\left\langle K^{\prime}(\Omega), \mathbf{V}\right\rangle=\int_{\Omega} L_{s} d \mathbf{x}-\int_{\Gamma} L(\mathbf{x})(V(\mathbf{x}) \cdot \mathbf{N}(\mathbf{x})) d \mathbf{a}(\mathbf{x}) .
$$

Since $L$ does not depend on $\Omega$, we obtain $L_{s}=0$ and we are done.

Putting all terms together, we obtain the complete expression of the Gâteaux derivative of $J$ :

$$
\left\langle J^{\prime}(\Omega), \mathbf{V}\right\rangle=-\int_{\Gamma}(A B L(\mathbf{x})+A H(\mathbf{x}, K(\Omega))+f(\mathbf{x}, \Omega))(\mathbf{V}(\mathbf{x}) \cdot \mathbf{N}(\mathbf{x})) d \mathbf{a}(\mathbf{x})
$$

with $B=\int_{\Omega} H_{K}(\mathbf{x}, K) d \mathbf{x}$.

The general case follows easily and is stated in the following theorem.

TheOREM 5.7. The Gâteaux derivative in the direction of $\mathbf{V}$ of the functional $J$ defined in (2.4) is

$$
\left\langle J^{\prime}(\Omega), \mathbf{V}\right\rangle=-\int_{\Gamma}\left(\sum_{i=1}^{m} A_{i} \sum_{j=1}^{l_{i}}\left(B_{i j} L_{i j}(\mathbf{x})\right)+\sum_{i=1}^{m}\left(A_{i} H_{i}\right)+f\right)(\mathbf{V}(\mathbf{x})) \cdot \mathbf{N}(\mathbf{x}) d \mathbf{a}(\mathbf{x}),
$$

where

$$
\begin{aligned}
A_{i} & =\int_{\Omega} f_{G_{i}}\left(x, G_{1}(\Omega), \ldots, G_{m}(\Omega)\right) d \mathbf{x}, \quad i=1 \ldots m \\
\text { and } \quad B_{i j} & =\int_{\Omega} H_{i K_{i j}}\left(x, K_{i 1}(\Omega), \ldots, K_{i l_{i}}(\Omega)\right) d \mathbf{x}, \quad i=1 \ldots m, \quad j=1 \ldots l_{i} .
\end{aligned}
$$

From the Gâteaux derivative of $J$, we deduce the corresponding evolution equation:

$$
\frac{\partial \Gamma}{\partial \tau}=\left[f(\Gamma)+\sum_{i=1}^{m} A_{i} H_{i}(\Gamma)+\sum_{i=1}^{m} A_{i}\left(\sum_{j=1}^{l_{i}} B_{i j} L_{i j}(\Gamma)\right)\right] \mathbf{N}
$$

which, as expected, is identical to (4.6). As far as the final result is concerned, the two methods of computation are equivalent.

5.4. Application. Let us now apply this method to the first example in section 2.3. The function $f$ is given by (2.8). The corresponding functions $G_{i}, H_{i}$ are given in section 2.3. We need the terms $A_{j}, j=1,2$ :

$$
\left\{\begin{array}{l}
A_{1}=-\int_{\Omega} \frac{1}{G_{2}} \varrho^{\prime}\left(I(\mathbf{x})-\frac{G_{1}}{G_{2}}\right) d \mathbf{x}=\frac{-1}{|\Omega|} \int_{\Omega} \varrho^{\prime}\left(I-\mu_{\Omega}\right) d \mathbf{x}, \\
A_{2}=\int_{\Omega} \frac{G_{1}}{\left(G_{2}\right)^{2}} \varrho^{\prime}\left(I(\mathbf{x})-\frac{G_{1}}{G_{2}}\right) d \mathbf{x}=\frac{\mu_{\Omega}}{|\Omega|} \int_{\Omega} \varrho^{\prime}\left(I-\mu_{\Omega}\right) d \mathbf{x} .
\end{array}\right.
$$

Since the terms $H_{i}$ are not region-dependent, the terms $B_{i j}$ are equal to zero. The velocity vector of the active contour is then the following:

$$
\frac{\partial \Gamma(\tau)}{\partial \tau}=\left[f-\frac{\left(I-\mu_{\Omega}\right)}{|\Omega|} \int_{\Omega} \varrho^{\prime}\left(I-\mu_{\Omega}\right) d \mathbf{x}\right] \mathbf{N} .
$$

In this example, the additional term coming from the region dependency of $f$ is equal to $\frac{\left(I-\mu_{\Omega}\right)}{|\Omega|} \int_{\Omega} \varrho^{\prime}\left(I-\mu_{\Omega}\right) d \mathbf{x}$. Note that in the particular case of $\varrho(r)=r^{2}$, this additional 
term is equal to zero $[6,7,20,21]$. However, in the general case, the additional term is not nul.

Let us apply this method to the second example in section 2.4. The function $f$ is a function of the variance given by (2.9). The corresponding functions $G_{i}, H_{i}, K_{i j}$, and $L_{i j}$ are also given in section 2.4. We need the terms $A_{j}, j=1,2$ :

$$
\left\{\begin{array}{l}
A_{1}=\int_{\Omega} \frac{1}{G_{2}} \varrho^{\prime}\left(\frac{G_{1}}{G_{2}}\right) d \mathbf{x}=\varrho^{\prime}\left(\sigma_{\Omega}^{2}\right), \\
A_{2}=-\int_{\Omega} \frac{G_{1}}{\left(G_{2}\right)^{2}} \varrho^{\prime}\left(\frac{G_{1}}{G_{2}}\right) d \mathbf{x}=-\sigma_{\Omega}^{2} \varrho^{\prime}\left(\sigma_{\Omega}^{2}\right) .
\end{array}\right.
$$

The terms $B_{i j}$ are given by

$$
\left\{\begin{array}{l}
B_{11}=\int_{\Omega} H_{1 K_{11}}\left(x, K_{11}, K_{12}\right)=-2 \frac{1}{\Omega \mid} \int_{\Omega}\left(I(\mathbf{x})-\mu_{\Omega}\right) d \mathbf{x}=0 \\
B_{12}=\int_{\Omega} H_{1 K_{12}}\left(x, K_{11}, K_{12}\right)=2 \frac{\mu_{\Omega}}{|\Omega|} \int_{\Omega}\left(I(\mathbf{x})-\mu_{\Omega}\right) d \mathbf{x}=0
\end{array}\right.
$$

We can then compute the velocity vector of the active contour from (5.5) and we find

$$
\frac{\partial \Gamma(\tau)}{\partial \tau}=\left[f+\varrho^{\prime}\left(\sigma_{\Omega}^{2}\right)\left(\left(I-\mu_{\Omega}\right)^{2}-\sigma_{\Omega}^{2}\right)\right] \mathbf{N} .
$$

In this simple example, we notice that the dependency of the function on the region induces an additional term in the evolution equation compared with the evolution equation obtained in the case where the function is region independent (equation (4.2)). This additional term is $\varrho^{\prime}\left(\sigma_{\Omega}^{2}\right)\left(\left(I(\mathbf{x})-\mu_{\Omega}\right)^{2}-\sigma_{\Omega}^{2}\right)$. It must be included in order to reach a true minimum of the criterion as proved in [33].

6. Matching histograms. A natural way of generalizing the use of statistical image features such as the mean and the variance of the intensity for image segmentation is to consider the full probability distribution of the feature of interest within the region, e.g., intensity, color, texture, etc., .... It turns out that in attempting to do so, one is naturally led to extend the criterion (2.4) to the case where the function $f$ depends on a continuous family of region criteria. Consider a function $\mathbf{h}: \mathbb{R}^{n} \rightarrow \mathbb{R}^{m}$ which describes the feature of interest. Suppose we have learnt the probability density function (pdf) of the feature $\mathbf{h}$ within the image region of interest, and let $q(\boldsymbol{\alpha})$ be this pdf. Given a region $\Omega$, we can estimate the pdf of the feature $\mathbf{h}$ through the use of the Parzen method [24]: let $p: \mathbb{R}^{m} \rightarrow \mathbb{R}^{+}$be the Parzen window, a smooth positive function whose integral is equal to 1 . For the sake of simplicity but without loss of generality, we assume that $p$ is an $m$-dimensional Gaussian with 0 -mean and variance $\sigma^{2}$, we note

$$
p(\boldsymbol{\alpha})=g_{\sigma}(\boldsymbol{\alpha})=\frac{1}{\left(2 \pi \sigma^{2}\right)^{m / 2}} \exp \left(-\frac{|\boldsymbol{\alpha}|^{2}}{2 \sigma^{2}}\right)
$$

and we define

$$
\hat{q}(\boldsymbol{\alpha}, \Omega)=\frac{1}{K(\Omega)} \int_{\Omega} g_{\sigma}(\mathbf{h}(\mathbf{x})-\boldsymbol{\alpha}) d \mathbf{x},
$$

where $\mathbf{h}(\mathbf{x})$ is the value of the feature of interest at the point $\mathbf{x}$ of $\Omega$ and $K$ is a normalizing constant, in general depending of $\Omega$, such that

$$
\int_{\mathbb{R}^{m}} \hat{q}(\boldsymbol{\alpha}, \Omega) d \boldsymbol{\alpha}=1 .
$$


Therefore

$$
K(\Omega)=\int_{\Omega} \int_{\mathbb{R}^{m}} g_{\sigma}(\mathbf{h}(\mathbf{x})-\boldsymbol{\alpha}) d \boldsymbol{\alpha} d \mathbf{x}=|\Omega|
$$

We next assume that we have a function $\varphi: \mathbb{R}^{+} \times \mathbb{R}^{m} \rightarrow \mathbb{R}^{+}$which allows us to compare two pdfs. This function is small if the pdfs are similar and large otherwise. It allows us to introduce the following functional which represents the "distance" between the two histograms:

$$
D(\Omega)=\int_{\mathbb{R}^{m}} \varphi(\hat{q}(\boldsymbol{\alpha}, \Omega), q(\boldsymbol{\alpha})) d \boldsymbol{\alpha} .
$$

The distance can be the square of the $L^{2}$ norm when

$$
\varphi(\hat{q}(\boldsymbol{\alpha}, \Omega), q(\boldsymbol{\alpha}))=(\hat{q}(\boldsymbol{\alpha}, \Omega)-q(\boldsymbol{\alpha}))^{2},
$$

or the commonly used Kullback-Leibler divergence when

$$
\varphi(\hat{q}(\boldsymbol{\alpha}, \Omega), q(\boldsymbol{\alpha}))=\frac{1}{2}\left(q(\boldsymbol{\alpha}) \log \frac{q(\boldsymbol{\alpha})}{\hat{q}(\boldsymbol{\alpha}, \Omega)}+\hat{q}(\boldsymbol{\alpha}, \Omega) \log \frac{\hat{q}(\boldsymbol{\alpha}, \Omega)}{q(\boldsymbol{\alpha})}\right),
$$

or the Hellinger distance when

$$
\varphi(\hat{q}(\boldsymbol{\alpha}, \Omega), q(\boldsymbol{\alpha}))=(\sqrt{\hat{q}(\boldsymbol{\alpha}, \Omega)}-\sqrt{q(\boldsymbol{\alpha})})^{2},
$$

or the nonsymmetric chi-2 comparison function when

$$
\varphi(\hat{q}(\boldsymbol{\alpha}, \Omega), q(\boldsymbol{\alpha}))=\frac{(\hat{q}(\boldsymbol{\alpha}, \Omega)-q(\boldsymbol{\alpha}))^{2}}{q(\boldsymbol{\alpha})} .
$$

A further generalization of the previous case is to consider second order histograms which describe the probability of having the value $\boldsymbol{\alpha}_{1}$ at pixel $\mathbf{x}$ and the value $\boldsymbol{\alpha}_{2}$ at pixel $\mathbf{x}+\boldsymbol{\delta}$, where $\boldsymbol{\delta}$ is a fixed (usually small) vector of $\mathbf{R}^{n}$. This has been used very much in computer vision for analyzing textures [28, 29]. The corresponding pdf, denoted $q_{\boldsymbol{\delta}}\left(\boldsymbol{\alpha}_{1}, \boldsymbol{\alpha}_{2}\right)$, can be estimated with the same Parzen window technique. We define

$$
\hat{q}_{\boldsymbol{\delta}}\left(\boldsymbol{\alpha}_{1}, \boldsymbol{\alpha}_{2}, \Omega\right)=\frac{1}{K_{\boldsymbol{\delta}}(\Omega)} \int_{\Omega} g_{\sigma}\left(\mathbf{h}(\mathbf{x})-\boldsymbol{\alpha}_{1}\right) g_{\sigma}\left(\mathbf{h}(\mathbf{x}+\boldsymbol{\delta})-\boldsymbol{\alpha}_{2}\right) d \mathbf{x} .
$$

The normalizing constant $K_{\boldsymbol{\delta}}(\Omega)$ is given by

$$
K_{\boldsymbol{\delta}}(\Omega)=\int_{\Omega} \int_{\mathbb{R}^{m} \times \mathbb{R}^{m}} g_{\sigma}\left(\mathbf{h}(\mathbf{x})-\boldsymbol{\alpha}_{1}\right) g_{\sigma}\left(\mathbf{h}(\mathbf{x}+\boldsymbol{\delta})-\boldsymbol{\alpha}_{2}\right) d \boldsymbol{\alpha}_{1} d \boldsymbol{\alpha}_{2} d \mathbf{x}=|\Omega| .
$$

We therefore define

$$
D_{\boldsymbol{\delta}}(\Omega)=\int_{\mathbb{R}^{m} \times \mathbb{R}^{m}} \varphi\left(\hat{q}_{\boldsymbol{\delta}}\left(\boldsymbol{\alpha}_{1}, \boldsymbol{\alpha}_{2}, \Omega\right), q_{\boldsymbol{\delta}}\left(\boldsymbol{\alpha}_{1}, \boldsymbol{\alpha}_{2}\right)\right) d \boldsymbol{\alpha}_{1} d \boldsymbol{\alpha}_{2}
$$

Using the tools developed in section 5, we compute the Gâteaux derivative of the functional $D$. We have the following theorem. 
Theorem 6.1. The Gâteaux derivative in the direction $\mathbf{V}$ of the functional $D$ defined in (6.1) is

$$
\left\langle D^{\prime}(\Omega), \mathbf{V}\right\rangle=-\frac{1}{|\Omega|} \int_{\Gamma}\left(\partial_{1} \varphi(\hat{q}(., \Omega), q(.)) * g_{\sigma}(\mathbf{h}(\mathbf{x}))-C(\Omega)\right)(\mathbf{V} \cdot \mathbf{N}) d \mathbf{a}(\mathbf{x}),
$$

where $C(\Omega)=\int_{\mathbb{R}^{m}} \partial_{1} \varphi(\hat{q}(\boldsymbol{\alpha}, \Omega), q(\boldsymbol{\alpha})) \hat{q}(\boldsymbol{\alpha}, \Omega) d \boldsymbol{\alpha}$.

Proof. By the definition of $D$ we have

$$
\left\langle D^{\prime}(\Omega), \mathbf{V}\right\rangle=\int_{\mathbb{R}^{m}}\left\langle(\varphi(\hat{q}(\boldsymbol{\alpha}, \Omega), q(\boldsymbol{\alpha})))^{\prime}, \mathbf{V}\right\rangle d \boldsymbol{\alpha} .
$$

Let us compute the Gâteaux derivative of $\varphi(\hat{q}(\boldsymbol{\alpha}, \Omega), q(\boldsymbol{\alpha}))$. We define

$$
\varphi(\hat{q}(\boldsymbol{\alpha}, \Omega), q(\boldsymbol{\alpha}))=f\left(G_{1}(\boldsymbol{\alpha}, \Omega), G_{2}(\Omega)\right)=\varphi\left(\frac{G_{1}(\boldsymbol{\alpha}, \Omega)}{G_{2}(\Omega)}, q(\boldsymbol{\alpha})\right),
$$

where

$$
\begin{aligned}
G_{1}(\boldsymbol{\alpha}, \Omega) & =\int_{\Omega} g_{\sigma}(\mathbf{h}(\mathbf{x})-\boldsymbol{\alpha}) d \mathbf{x} \text { with } H_{1}(\boldsymbol{\alpha}, \mathbf{x})=g_{\sigma}(\mathbf{h}(\mathbf{x})-\boldsymbol{\alpha}), \\
G_{2}(\Omega) & =|\Omega|=\int_{\Omega} d \mathbf{x} .
\end{aligned}
$$

We obtain

$$
\begin{aligned}
\left\langle f^{\prime}, \mathbf{V}\right\rangle & =f_{G_{1}}\left\langle G_{1}^{\prime}, \mathbf{V}\right\rangle+f_{G_{2}}\left\langle G_{2}^{\prime}, \mathbf{V}\right\rangle \\
& =\frac{\partial_{1} \varphi(\hat{q}(\boldsymbol{\alpha}, \Omega), q(\boldsymbol{\alpha}))}{|\Omega|}\left(\left\langle G_{1}^{\prime}, \mathbf{V}\right\rangle-\hat{q}(\boldsymbol{\alpha}, \Omega)\left\langle G_{2}^{\prime}, \mathbf{V}\right\rangle\right)
\end{aligned}
$$

and, using Theorem 5.5,

$$
\left\langle f^{\prime}, \mathbf{V}\right\rangle=-\frac{\partial_{1} \varphi(\hat{q}(\boldsymbol{\alpha}, \Omega), q(\boldsymbol{\alpha}))}{|\Omega|} \int_{\Gamma}\left(g_{\sigma}(\mathbf{h}(\mathbf{x})-\boldsymbol{\alpha})-\hat{q}(\boldsymbol{\alpha}, \Omega)\right)(\mathbf{V} \cdot \mathbf{N}) d \mathbf{a}(\mathbf{x}) .
$$

Plugging this result into the expression of $\left\langle D^{\prime}(\Omega), \mathbf{V}\right\rangle$ and swapping the order of integration, we obtain

$$
\begin{aligned}
\left\langle D^{\prime}(\Omega), \mathbf{V}\right\rangle=-\frac{1}{|\Omega|} \int_{\Gamma}\left(\int_{\mathbb{R}^{m}} g_{\sigma}(\mathbf{h}(\mathbf{x})-\boldsymbol{\alpha}) \partial_{1} \varphi(\hat{q}(\boldsymbol{\alpha}, \Omega), q(\boldsymbol{\alpha})) d \boldsymbol{\alpha}\right. \\
\left.\quad-\int_{\mathbb{R}^{m}} \partial_{1} \varphi(\hat{q}(\boldsymbol{\alpha}, \Omega), q(\boldsymbol{\alpha})) \hat{q}(\boldsymbol{\alpha}, \Omega) d \boldsymbol{\alpha}\right)(\mathbf{V} \cdot \mathbf{N}) d \mathbf{a}(\mathbf{x}) .
\end{aligned}
$$

The first integral on the right-hand side is the convolution $\partial_{1} \varphi(\hat{q}(., \Omega), q()). * g_{\sigma}$ of the function $\partial_{1} \varphi(\hat{q}(., \Omega), q()):. \mathbb{R}^{m} \rightarrow \mathbb{R}$ with the function $g_{\sigma}$. The final result is

$$
\left\langle D^{\prime}(\Omega), \mathbf{V}\right\rangle=-\frac{1}{|\Omega|} \int_{\Gamma}\left(\partial_{1} \varphi(\hat{q}(., \Omega), q(.)) * g_{\sigma}(\mathbf{h}(\mathbf{x}))-C(\Omega)\right)(\mathbf{V} \cdot \mathbf{N}) d \mathbf{a}(\mathbf{x}),
$$

where $C(\Omega)=\int_{\mathbb{R}^{m}} \partial_{1} \varphi(\hat{q}(\boldsymbol{\alpha}, \Omega), q(\boldsymbol{\alpha})) \hat{q}(\boldsymbol{\alpha}, \Omega) d \boldsymbol{\alpha}$.

This solves the question of first order histograms. For second order histograms we have the following theorem. 
TheOrem 6.2. The Gâteaux derivative in the direction $\mathbf{V}$ of the functional $D_{\boldsymbol{\delta}}$ defined in (6.2) is

$$
\begin{gathered}
\left\langle D_{\boldsymbol{\delta}}^{\prime}(\Omega), \mathbf{V}\right\rangle=-\frac{1}{|\Omega|} \int_{\Gamma}\left(\partial_{1} \varphi\left(\hat{q}_{\boldsymbol{\delta}}(., ., \Omega), q(., .)\right) *\left(g_{\sigma} \otimes g_{\sigma}\right)(\mathbf{h}(\mathbf{x}), \mathbf{h}(\mathbf{x}+\boldsymbol{\delta}))\right. \\
\left.-C_{\boldsymbol{\delta}}(\Omega)\right)(\mathbf{V} \cdot \mathbf{N}) d \mathbf{a}(\mathbf{x})
\end{gathered}
$$

where $C_{\boldsymbol{\delta}}(\Omega)=\int_{\mathbb{R}^{m} \times \mathbb{R}^{m}} \partial_{1} \varphi\left(\hat{q}_{\boldsymbol{\delta}}\left(\boldsymbol{\alpha}_{1}, \boldsymbol{\alpha}_{2}, \Omega\right), q\left(\boldsymbol{\alpha}_{1}, \boldsymbol{\alpha}_{2}\right)\right) \hat{q}_{\boldsymbol{\delta}}\left(\boldsymbol{\alpha}_{1}, \boldsymbol{\alpha}_{2}, \Omega\right) d \boldsymbol{\alpha}_{1} d \boldsymbol{\alpha}_{2}$, and $g_{\sigma} \otimes$ $g_{\sigma}\left(\boldsymbol{\alpha}_{1}, \boldsymbol{\alpha}_{2}\right)=g_{\sigma}\left(\boldsymbol{\alpha}_{1}\right) g_{\sigma}\left(\boldsymbol{\alpha}_{2}\right)$.

Proof. The proof is identical to that of Theorem 6.1.

7. Color histograms: Segmentation of regions in video sequences. This work has been motivated by $[12,8]$ where the tracking algorithms take advantage of statistical color distributions. We propose here to use active contours in order to fit exactly the shape of the object to be segmented. We consider a video sequence where each frame is represented by the color function $\mathbf{h}: \mathbb{R}^{2} \rightarrow \mathbb{R}^{2}$. The color space used is $(H, V)$, where $H$ stands for the hue and $V$ for the value. ${ }^{1}$ The goal is to segment a reference region, given in the previous image of the sequence, into the current one by minimizing the distance between the reference histogram $q$ of the region in the previous image and the estimated histogram $\hat{q}$ in the current frame. From an initial curve chosen by the user in the current frame, we want to make an active contour evolve towards the region in the current frame whose histogram is closest to the reference histogram of the previous frame.

In order to introduce a competition between the region of interest and the background region, we also consider the complement $\Omega^{c}$ of the region $\Omega$ of interest. They share the same boundary, $\Gamma$, but with normals pointing in opposite directions. We denote $q^{c}$ the reference histogram of $\Omega^{c}$, and we look for the region $\Omega$ which minimizes the following criterion: ${ }^{2}$

$$
J(\Omega)=D(\Omega)+D\left(\Omega^{c}\right)+\lambda \int_{\Gamma} d s
$$

In this criterion, the first two terms are region functionals while the last one is a boundary functionals. The last one minimizes the curve length and is a regularization term weighted by the positive parameter $\lambda$. We have, of course,

$$
\begin{aligned}
D(\Omega) & =\int_{\mathbb{R}^{2}} \varphi(\hat{q}(\boldsymbol{\alpha}, \Omega), q(\boldsymbol{\alpha})) d \boldsymbol{\alpha}, \\
D\left(\Omega^{c}\right) & =\int_{\mathbb{R}^{2}} \varphi\left(\hat{q}\left(\boldsymbol{\alpha}, \Omega^{c}\right), q(\boldsymbol{\alpha})\right) d \boldsymbol{\alpha} .
\end{aligned}
$$

Computation of the Gâteaux derivative. A straightforward application of Theorem 6.1 yields

$$
\left\langle D^{\prime}(\Omega), \mathbf{V}\right\rangle=-\frac{1}{|\Omega|} \int_{\Gamma}\left(\partial_{1} \varphi(\hat{q}(., \Omega), q(.)) * g_{\sigma}(\mathbf{h}(\mathbf{x}))-C(\Omega)\right)(\mathbf{V} \cdot \mathbf{N}) d s
$$

\footnotetext{
${ }^{1}$ We ignore the saturation to avoid the curse of dimensionality.

${ }^{2}$ The results are even better if we introduce the region area in the criterion by minimizing $D(\Omega)|\Omega|+D\left(\Omega^{c}\right)\left|\Omega^{c}\right|+\lambda \int_{\Gamma} d s$.
} 
with

$$
C(\Omega)=\int_{\mathbb{R}^{m}} \partial_{1} \varphi(\hat{q}(\boldsymbol{\alpha}, \Omega), q(\boldsymbol{\alpha})) \hat{q}(\boldsymbol{\alpha}, \Omega) d \boldsymbol{\alpha} .
$$

Similar results hold for $\Omega^{c}$ :

$$
\left\langle D^{\prime}\left(\Omega^{c}\right), \mathbf{V}\right\rangle=\frac{1}{\left|\Omega^{c}\right|} \int_{\Gamma}\left(\partial_{1} \varphi\left(\hat{q}\left(., \Omega^{c}\right), q^{c}(.)\right) * g_{\sigma}(\mathbf{h}(\mathbf{x}))-C\left(\Omega^{c}\right)\right)(\mathbf{V} \cdot \mathbf{N}) d s,
$$

with

$$
C\left(\Omega^{c}\right)=\int_{\mathbb{R}^{m}} \partial_{1} \varphi\left(\hat{q}\left(\boldsymbol{\alpha}, \Omega^{c}\right), q^{c}(\boldsymbol{\alpha})\right) \hat{q}\left(\boldsymbol{\alpha}, \Omega^{c}\right) d \boldsymbol{\alpha} .
$$

Computation of the evolution equation of an active contour. It is well known that the minimization of the curve length leads to the Euclidean curve shortening flow $\lambda \kappa$ $[4,35]$. Then, from the previous derivatives, we can deduce the evolution of an active contour that will evolve towards a minimum of the criterion $J_{n}$ defined in (7.1). We find the evolution equation

$$
\frac{\partial \Gamma(\tau)}{\partial \tau}=F \mathbf{N}
$$

with

$$
\begin{aligned}
F=\lambda \kappa & +\frac{1}{|\Omega|}\left(\partial_{1} \varphi(\hat{q}(., \Omega), q(.)) * g_{\sigma}(\mathbf{h}(\mathbf{x}))-C(\Omega)\right) \\
& -\frac{1}{\left|\Omega^{c}\right|}\left(\partial_{1} \varphi\left(\hat{q}\left(., \Omega^{c}\right), q^{c}(.)\right) * g_{\sigma}(\mathbf{h}(\mathbf{x}))-C\left(\Omega^{c}\right)\right),
\end{aligned}
$$

where $\kappa$ is the curvature of $\Gamma$ and $C(\Omega), C\left(\Omega^{c}\right)$ are given by (7.2) and (7.3), respectively.

Let us take the example of the Hellinger distance, where $\partial_{1} \varphi(r, \boldsymbol{\alpha})=(\sqrt{r}-$ $\sqrt{q(\boldsymbol{\alpha})}) / \sqrt{r}$. We find for the velocity vector

$$
\begin{aligned}
F=\lambda \kappa & +\frac{1}{|\Omega|}\left(\frac{(\sqrt{\hat{q}(., \Omega)}-\sqrt{q(.)})}{\sqrt{\hat{q}(., \Omega)}} * g_{\sigma}(\mathbf{h}(\mathbf{x}))-C(\Omega)\right) \\
& -\frac{1}{\left|\Omega^{c}\right|}\left(\frac{\left(\sqrt{\hat{q}\left(., \Omega^{c}\right)}-\sqrt{q^{c}(.)}\right)}{\sqrt{\hat{q}\left(., \Omega^{c}\right)}} * g_{\sigma}(\mathbf{h}(\mathbf{x}))-C\left(\Omega^{c}\right)\right) .
\end{aligned}
$$

And for the chi-2 comparison function where $\partial_{1} \varphi(r, \boldsymbol{\alpha})=2(r-q(\boldsymbol{\alpha})) / q(\boldsymbol{\alpha})$, we find

$$
\begin{aligned}
F=\lambda \kappa & +\frac{2}{|\Omega|}\left(\frac{(\hat{q}(., \Omega)-q(.))}{q(.)} * g_{\sigma}(\mathbf{h}(\mathbf{x}))-C(\Omega)\right) \\
& -\frac{2}{\left|\Omega^{c}\right|}\left(\frac{\left(\hat{q}\left(., \Omega^{c}\right)-q^{c}(.)\right)}{q^{c}(.)} * g_{\sigma}(\mathbf{h}(\mathbf{x}))-C\left(\Omega^{c}\right)\right) .
\end{aligned}
$$

In the velocity, the convolution term allows us to compare locally the reference histogram to the current histogram. 
7.1. Implementation. As far as the numerical implementation is concerned, we can model the active contour with either an explicit parameterization (Lagrangian formulation) or an implicit one (Eulerian formulation). See [23] for an interesting comparison between the two methods. Another interesting review may be found in [36].

Here, we use the level set method approach first proposed by Osher and Sethian [37] and applied this to active contours in [3]. The key idea of the level set method is to introduce an auxiliary function $U(\mathbf{x}, \tau)$ such that $\Gamma(\tau)$ is the zero level set of $U$. The function $U$ is often chosen to be the signed distance function of $\Gamma(\tau)$ which satisfies

$$
\Gamma(\tau)=\{\mathbf{x} \mid U(\mathbf{x}, \tau)=0\} \quad \text { and } \quad|\nabla U|=1 .
$$

This Eulerian formulation presents several advantages [47]. First, the curve $U$ may break or merge as the function $U$ evolves, and topological changes are thus easily handled. Second, the evolving function $U(\mathbf{x}, \tau)$ always remains a function allowing efficient numerical schemes. Third, the geometric properties of the curve, like the curvature $\kappa$ and the normal vector field $\mathbf{N}$, can be estimated directly from the level set function:

$$
\kappa=\operatorname{div}\left(\frac{\nabla U}{|\nabla U|}\right) \quad \text { and } \quad \mathbf{N}=-\frac{\nabla U}{|\nabla U|} .
$$

The evolution equation (7.4) then becomes

$$
\frac{\partial U(\tau)}{\partial \tau}=F|\nabla U| .
$$

The velocity function $F$ is computed only on the curve $\Gamma(\tau)$, but we can extend its expression to the whole image domain $\Omega$. To implement the level set method, solutions must be found to circumvent problems coming from the fact that the signed distance function $U$ is not a solution of the PDE (7.5); see [26] for details. In our work, the function $U$ is re-initialized so that it remains a distance function. Details on the re-initialization equation are provided in $[1,19]$.

In order to improve numerical efficiency, we compute the equation in a narrow band enclosing the 0 level of the level set function $[47,48]$. We also use multiresolution techniques by making the active contour evolve first in a low resolution image. The final contour obtained for this reduced image is then used as an initial curve for the real size image. Another possibility for increasing efficiency would be the use of accurate operator splitting (AOS) schemes [50].

7.2. Experimental results. Experimental results have been obtained on the sequence "Erik" from the European group COST211. Experiments are conducted using the chi-2 comparison function with $\varphi(r, \alpha)=(r-q(\boldsymbol{\alpha}))^{2} / q(\boldsymbol{\alpha})$ and $\partial_{1} \varphi(r, \boldsymbol{\alpha})=$ $2(r-q(\boldsymbol{\alpha})) / q(\boldsymbol{\alpha})$.

The region of interest is the face. We assume that it has been segmented in the first image as shown in Figure 1(a). The first two reference histograms are computed. The two reference histograms are also given Figure 1(b) for the background reference histogram $q^{c}$ and Figure 1(c) for the object reference histogram, $q$. For a given region $\Omega$ and for a point $\boldsymbol{\alpha}=\left[\alpha_{1}, \alpha_{2}\right]^{T}$, the function $\hat{q}(\boldsymbol{\alpha}, \Omega)$ represents the probability to obtain $H(\mathbf{x})=\alpha_{1}$ and $V(\mathbf{x})=\alpha_{2}$ for $\mathbf{x}$ belonging to the region $\Omega$.

Then, using the two reference histograms of the previous frame, we make the active contour evolve using (7.4) in the current frame. The initial curve is chosen 


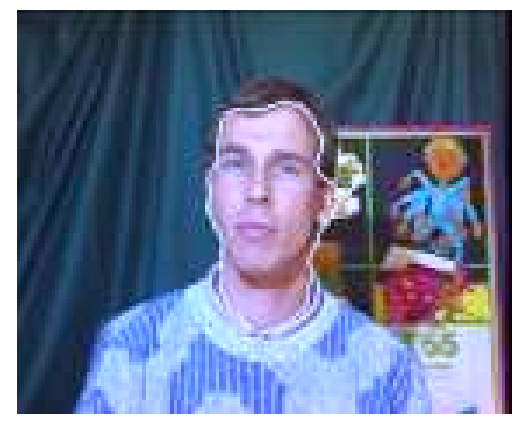

a. Reference segmentation

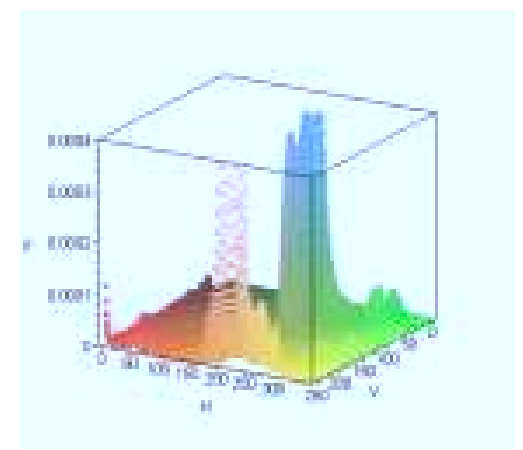

b. Background reference histogram

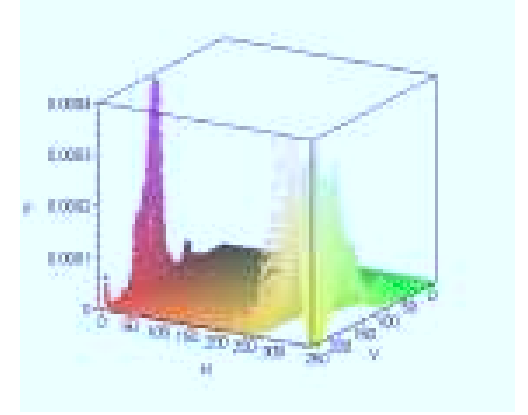

c. Object reference histogram

FIG. 1. The reference segmentation of the previous frame (a), the corresponding background reference histogram $q^{c}$ (b), and the corresponding object reference histogram $q$ (c).

to be a circle. The evolution of the active contour in the current frame is shown in Figure 2. We notice that the final contour in Figure 2(c) nicely describes the region of interest, and the face is accurately segmented. We can also visualize the evolution of the object histogram, $\hat{q}(\boldsymbol{\alpha}, \Omega)$, during the propagation of the active contour. The final object histogram given in Figure 2(d) can be compared to the reference object histogram in Figure 1(c), showing an efficient minimization of the distance between the two histograms.

8. Conclusion. In this article we have clarified the relationships between the boundary and region functionals that arise naturally in several image processing tasks. We have shown that one can go from one to the other by solving Poisson's equation with Dirichlet conditions or Helmholtz's equation with Neumann conditions.

We have then concentrated on the problem of finding local minima of a large class of region functionals. By first transforming them into boundary functionals and applying methods from the calculus of variations we have computed the corresponding Gâteaux derivatives and constructed a velocity field on the region boundary. This field defines a PDE whose solution, for a given initial boundary, generates a oneparameter family of regions which, in practice, converges toward a local minimum of the functional. The problem of the existence and uniqueness of a solution to this PDE has not been addressed.

Changing our point of view, we have then rederived the same equations in a simpler and more natural way, i.e., without going through the trouble of turning region integrals into boundary integrals; this is achieved by applying methods of shape derivation $[49,22]$. 


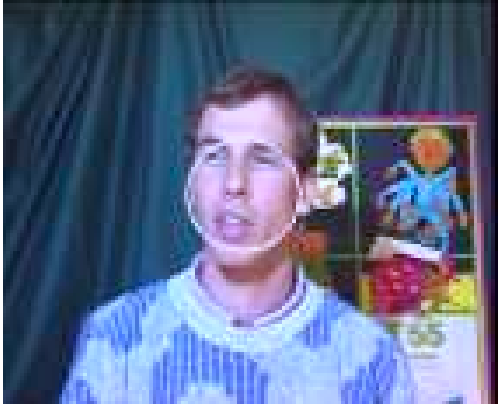

a. Initial contour
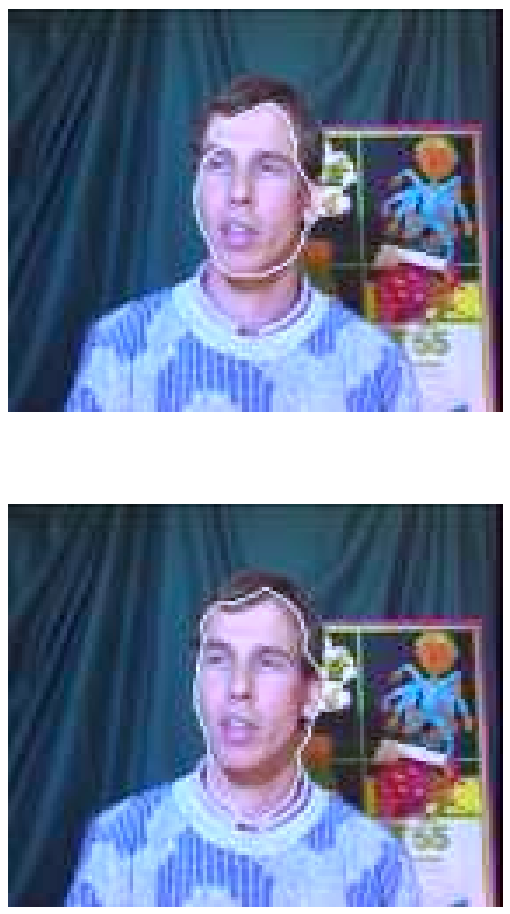

c. Final Contour

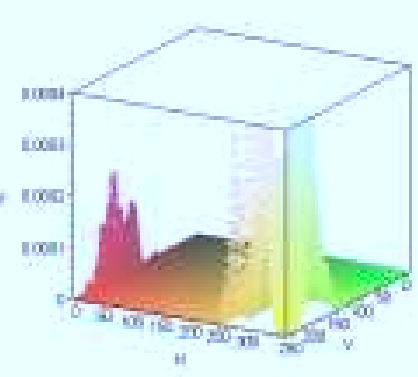

b. Initial histogram $\hat{q}(\boldsymbol{\alpha}, \Omega)$
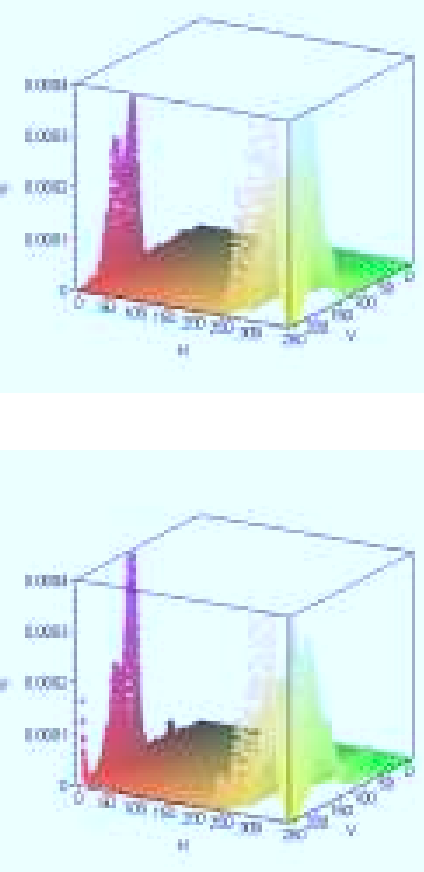

d. Final histogram $\hat{q}(\boldsymbol{\alpha}, \Omega)$

FIG. 2. Evolution of the region histogram $\hat{q}(\boldsymbol{\alpha}, \Omega)$ of the current frame during the evolution of the active contour.

We have then turned our attention to a new class of region-based functionals by considering histograms of image features. The shape derivation tools have allowed us to easily derive the velocity field that defines the evolution of the region boundary.

The final part of the paper has been devoted to an application of the previous methods to the problem of region segmentation with a given color histogram in a sequence of images. Our experimental results show that the technique has indeed some interesting potentials.

Acknowledgments. We thank Rachid Deriche for his helpful comments on an early draft of this document. We also thank Gerardo Hermosillo for providing us with his software package for the robust estimation of image histograms. 


\section{REFERENCES}

[1] G. Aubert And J.-F. Aujol, Signed Distance Functions and Viscosity Solutions of Discontinuous Hamilton-Jacobi Equations, manuscript, 2002.

[2] H. Brezis, Analyse fonctionnelle. Théorie et applications, Masson, Paris, 1983.

[3] V. Caselles, F. Catte, T. Coll, and F. Dibos, A geometric model for active contours, Numer. Math., 66 (1993), pp. 1-31.

[4] V. Caselles, R. Kimmel, and G. Sapiro, Geodesic active contours, Internat. J. Comput. Vision, 22 (1997), pp. 61-79.

[5] A. Chakraborty, L. Staib, and J. Duncan, Deformable boundary finding in medical images by integrating gradient and region information, IEEE Trans. Medical Imaging, 15 (1996), pp. 859-870.

[6] T. Chan And L. Vese, An active contour model without edges, in Scale-Space Theories in Computer Vision, Lecture Notes in Comput. Sci. 1682, Springer-Verlag, Berlin, 1999.

[7] T. Chan and L. Vese, Active contours without edges, IEEE Trans. Image Processing, 10 (2001), pp. 266-277.

[8] H. Chen and T. Liu, Trust-region methods for real-time tracking, in Proceedings of the International Conference on Computer Vision, Vol. 2, Vancouver, Canada, 2001, pp. 717-722.

[9] C. Chesnaud, P. RéfréGier, And V. Boulet, Statistical region snake-based segmentation adapted to different physical noise models, IEEE Trans. Pattern Analysis Machine Intelligence, 21 (1999), pp. 1145-1156.

[10] L. Cohen, On active contour models and balloons, Comput. Vision Graphics Image Process., 53 (1991), pp. 211-218

[11] L. Cohen, E. Bardinet, And N. Ayache, Surface reconstruction using active contour models, in Proceedings of the SPIE Conference on Geometric Methods in Computer Vision, San Diego, CA, 1993.

[12] D. Comaniciu, V. Ramesh, and P. Meer, Real-time tracking of non-rigid objects using mean shift, in Computer Vision and Pattern Recognition, Vol. 2, IEEE Press, Piscataway, NJ, 2000, pp. 142-149.

[13] R. Dautray and J.-L. Lions, Mathematical Analysis and Numerical Methods for Science and Technology. Vol. 1. Physical Origins and Classical Methods, Springer-Verlag, Berlin, 1990.

[14] R. Dautray and J.-L. Lions, Mathematical Analysis and Numerical Methods for Science and Technology. Vol. 2. Functional and Variational Methods, Springer-Verlag, Berlin, 1988.

[15] R. Dautray and J.-L. Lions, Mathematical Analysis and Numerical Methods for Science and Technology. Vol. 3. Spectral Theory and Applications, Springer-Verlag, Berlin, 1990.

[16] R. Dautray and J.-L. Lions, Mathematical Analysis and Numerical Methods for Science and Technology. Vol. 4. Integral Equations and Numerical Methods, Springer-Verlag, Berlin, 1990.

[17] R. Dautray and J.-L. Lions, Mathematical Analysis and Numerical Methods for Science and Technology. Vol. 5. Evolution Problems. I, Springer-Verlag, Berlin, 1992.

[18] R. Dautray and J.-L. Lions, Mathematical Analysis and Numerical Methods for Science and Technology. Vol. 6. Evolution Problems. II, Springer-Verlag, Berlin, 1993.

[19] E. Debreuve, Segmentation par contours actifs en imagerie médicale dynamique : application en cardiologie nucléaire, Ph.D. thesis, University of Nice-Sophia Antipolis, France, 2000.

[20] E. Debreuve, M. Barlaud, G. Aubert, and J. Darcourt, Space time segmentation using level set active contours applied to myocardial gated SPECT, in Proceedings of the IEEE Medical Imaging Conference, Seattle USA, 1999.

[21] E. Debreuve, M. Barlaud, G. Aubert, and J. Darcourt, Space time segmentation using level set active contours applied to myocardial gated SPECT, IEEE Trans. Medical Imaging, 20 (2001), pp. 643-659.

[22] M. C. Delfour and J.-P. Zolésio, Shapes and Geometries: Analysis, Differential Calculus, and Optimization, Adv. Des. Control 4, SIAM, Philadelphia, 2001.

[23] H. Delingette And J. Montagnat, Topology and shape constraints on parametric active contours, Computer Vision and Image Understanding, 83 (2001), pp. 140-171.

[24] R. Duda And P. Hart, Pattern Classification and Scene Analysis, John Wiley, New York, 1973.

[25] L. Evans, Partial Differential Equations, Graduate Studies in Mathematics 19, AMS, Providence, RI, 1998.

[26] J. Gomes and O. Faugeras, Reconciling distance functions and level sets, J. Visual Communication and Image Representation, 11 (2000), pp. 209-223.

[27] J. HADAmARD, Mémoire sur un problème d'analyse relatif à l'équilibre des plaques élastiques encastrées, mémoire des savants étrangers, CNRS, Paris, 1968.

[28] R. Haralick and L. Shapiro, Computer and Robot Vision, Vol. 1, Addison-Wesley, Reading, 
MA, 1992.

[29] R. Haralick and L. Shapiro, Computer and Robot Vision, Vol. 2, Addison-Wesley, Reading MA, 1993.

[30] S. Jehan-Besson, M. Barlaud, and G. Aubert, Region-based active contours for video object segmentation with camera compensation, in Proceedings of the IEEE International Conference on Image Processing, Thessaloniki, Greece, 2001.

[31] S. Jehan-Besson, M. Barlaud, and G. Aubert, Video object segmentation using eulerian region-based active contours, in Proceedings of the IEEE International Conference on Computer Vision, Vancouver, Canada, 2001, pp. 353-361.

[32] S. Jehan-Besson, M. Barlaud, And G. Aubert, A 3-step algorithm using region-based active contours for video objects detection, EURASIP J. Appl. Signal Process., 6 (2002), pp. $572-581$.

[33] S. Jehan-Besson, M. Barlaud, and G. Aubert, DREAM ${ }^{2} S$ : Deformable regions driven by an eulerian accurate minimization method for image and video segmentation, application to face detection in color video sequences, in Computer Vision - ECCV 2002, Part 3, Lecture Notes in Comput. Sci. 2352, A. Heyden, G. Sparr, M. Nielsen, and P. Johansen, eds., Springer, Berlin, 2002, pp. 365-380.

[34] M. Kass, A. Witkin, And D. Terzopoulos, Snakes: Active contour models, Internat. J. Comput. Vision, 1 (1988), pp. 321-332.

[35] S. Kischenassamy, A. Kumar, P. Olver, A. Tannenbaum, and A. Yezzi, Conformal curvature flows: From phase transitions to active vision, Arch. Rational Mech. Anal., 134 (1996), pp. 275-301.

[36] J. Montagnat, H. Delingette, and N. Ayache, A review of deformable surfaces: Topology, geometry and deformation, Image and Vision Computing, 19 (2001), pp. 1023-1040.

[37] S. Osher And J. Sethian, Fronts propagating with curvature-dependent speed: Algorithms based on Hamilton-Jacobi formulation, J. Comput. Phys., 79 (1988), pp. 12-49.

[38] N. Paragios And R. Deriche, Geodesic active regions for motion estimation and tracking, in Proceedings of the IEEE International Conference on Computer Vision, Corfu Greece, 1999, pp. 688-694.

[39] N. Paragios And R. Deriche, Coupled geodesic active regions for image segmentation: A level set approach, in Proceedings of the European Conference in Computer Vision, Dublin, Ireland, 2000.

[40] N. Paragios and R. Deriche, Geodesic active regions: A new paradigm to deal with frame partition problems in computer vision, J. Visual Communication and Image Representation, 13 (2002), pp. 249-268.

[41] N. Paragios And R. Deriche, Geodesic active regions and level set methods for supervised texture segmentation, Internat. J. Comput. Vision, 46 (2002), p. 223.

[42] P.-A. Raviart And J.-M. Thomas, Introduction à l'analyse numérique des équations aux dérivées partielles, Masson, Paris, 1983.

[43] R. Ronfard, Region-based strategies for active contour models, Internat. J. Comput. Vision, 13 (1994), pp. 229-251.

[44] C. Samson, L. Blanc-Féraud, G. Aubert, and J. Zerubia, A level set model for image classification, in Scale-Space Theories in Computer Vision, Lecture Notes in Comput. Sci. 1682, Springer, Berlin, 1999.

[45] C. Samson, L. Blanc-Féraud, G. Aubert, and J. Zerubia, A level set model for image classification, Internat. J. Comput. Vision, 40 (2000), pp. 187-197.

[46] C. SChNöRR, Computation of discontinuous optical flow by domain decomposition and shape optimization, Internat. J. Comput. Vision, 8 (1992), pp. 153-165.

[47] J. Sethian, Level Set Methods, Cambridge University Press, Cambridge, UK, 1996.

[48] J. Sethian, Level Set Methods and Fast Marching Methods: Evolving Interfaces in Computational Geometry, Fluid Mechanics, Computer Vision, and Materials Sciences, Cambridge Monogr. Appl. Comput. Math., Cambridge University Press, Cambridge, UK, 1999.

[49] J. Sokolowski And J.-P. ZolÉsio, Introduction to shape optimization. Shape sensitivity analysis., Springer Ser. Comput. Math. 16, Springer-Verlag, Berlin, 1992.

[50] J. Weickert, B. ter HaAr Romeny, And M. Viergever, Efficient and reliable schemes for nonlinear diffusion filtering, IEEE Trans. Image Process., 7 (1998), pp. 398-410.

[51] A. YezZI, A. Tsai, AND A. Willsky, A statistical approach to snakes for bimodal and trimodal imagery, in Proceedings of the IEEE International Conference on Image Processing, Kobe Japan, 1999.

[52] S. ZHU AND A. YullLe, Region competition: Unifying snakes, region growing, and bayes/MDL for multiband image segmentation, IEEE Trans. Pattern Analysis Machine Intelligence, 18 (1996), pp. 884-900. 